Geometry 85 Topology

Volume 7 (2003) 91-153

Published: 17 February 2003

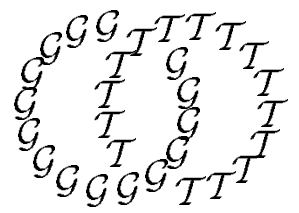

\title{
The sigma orientation for analytic circle-equivariant elliptic cohomology
}

\author{
Matthew Ando \\ Department of Mathematics \\ University of Illinois at Urbana-Champaign \\ Urbana IL 61801, USA \\ Email: mando@math.uiuc.edu
}

\begin{abstract}
We construct a canonical Thom isomorphism in Grojnowski's equivariant elliptic cohomology, for virtual $\mathbb{T}$-oriented $\mathbb{T}$-equivariant spin bundles with vanishing Borel-equivariant second Chern class, which is natural under pull-back of vector bundles and exponential under Whitney sum. It extends in the complexanalytic case the non-equivariant sigma orientation of Hopkins, Strickland, and the author. The construction relates the sigma orientation to the representation theory of loop groups and Looijenga's weighted projective space, and sheds light even on the non-equivariant case. Rigidity theorems of Witten-Bott-Taubes including generalizations by Kefeng Liu follow.
\end{abstract}

\section{AMS Classification numbers Primary: 55 N34}

Secondary: $55 \mathrm{~N} 22,57 \mathrm{R} 91$

Keywords: Sigma orientation, equivariant elliptic cohomolgy, rigidity

Proposed: Haynes Miller

Seconded: Ralph Cohen, Gunnar Carlsson
Received: 1 February 2002

Revised: 18 October 2002

(C) Geometry $8 \mathcal{G}$ Topology Publications 


\section{Introduction}

Let $E$ be an even periodic, homotopy commutative ring spectrum, let $C$ be an elliptic curve over $S_{E}=\operatorname{spec} \pi_{0} E$, and let $t$ be an isomorphism of formal groups

$$
t: \widehat{C} \cong \operatorname{spf} E^{0}\left(\mathbb{C} P^{\infty}\right),
$$

so that $(E, C, t)$ is an elliptic spectrum in the sense of [13, 2]. In [2], Hopkins, Strickland, and the author construct a canonical map of homotopy commutative ring spectra

$$
\sigma(E, C, t): M U\langle 6\rangle \rightarrow E
$$

called the sigma orientation; it is conjectured in [13] that this map is the restriction to $M U\langle 6\rangle$ of a similar map $M O\langle 8\rangle \rightarrow E$.

Let $\mathbb{T}$ be the circle group. We expect that there is an equivariant elliptic spectrum $\left(E_{\mathbb{T}}, C, t\right)$ extending the nonequivariant elliptic spectrum $(E, C, t)$, and that the sigma orientation extends to a multiplicative map of $\mathbb{T}$-equivariant spectra

$$
\sigma_{\mathbb{T}}\left(E_{\mathbb{T}}, C, t\right):(\mathbb{T} \text {-equivariant } M O\langle 8\rangle) \rightarrow E_{\mathbb{T}} .
$$

Note however that the construction of $\sigma_{\mathbb{T}}$ requires us among other things to say what $\mathbb{T}$-spectra we have in mind for the domain and codomain.

If $V$ is a virtual vector bundle over a space $X$, let $X^{V}$ denote its Thom spectrum. In principle, giving a map $\sigma_{\mathbb{T}}$ as in (1.1) should be equivalent to specifying, for each virtual $\mathbb{T}-B O\langle 8\rangle$ vector bundle (whatever that means) a trivialization $\gamma(V)$ of $E_{\mathbb{T}}\left(X^{V}\right)$ as an $E_{\mathbb{T}}(X)$-module; the trivialization should be stable, exponential, and natural as $V / X$ ranges over the virtual $\mathbb{T}-B O\langle 8\rangle$ vector bundles.

The trivializations $\gamma(V)$ should be compatible with the nonequivariant sigma orientation in the following way. If $X$ is a $\mathbb{T}$-space, then $X_{\mathbb{T}}$ will denote the Borel construction $E \mathbb{T} \times_{\mathbb{T}} X$; if $V$ is a (virtual) $\mathbb{T}$-vector bundle over $X$, then $V_{\mathbb{T}}$ will denote the corresponding (virtual) bundle over $X_{\mathbb{T}}$. A $\mathbb{T}-B O\langle 8\rangle$ structure on a $\mathbb{T}$-bundle should at least give a $B O\langle 8\rangle$ structure to $V_{\mathbb{T}}$. One expects that the equivariant extension $E_{\mathbb{T}}$ of an elliptic cohomology theory $E$ comes with a completion isomorphism

$$
E_{\mathbb{T}}(X)^{\wedge} \cong E\left(X_{\mathbb{T}}\right)
$$

and in particular

$$
E_{\mathbb{T}}\left(X^{V}\right)^{\wedge} \cong E\left(\left(X_{\mathbb{T}}\right)^{V_{\mathbb{T}}}\right)
$$


The desired compatibility is that this isomorphism carries $\gamma(V)$ to the sigma orientation of $V_{\mathbb{T}}$.

In this paper we take $E_{\mathbb{T}}$ to be the complex-analytic equivariant elliptic cohomology of Grojnowski. Let $\Lambda \subset \mathbb{C}$ be a lattice in the complex plane, and let $C$ be the analytic variety $C=\mathbb{C} / \Lambda$. Grojnowski constructs a functor $E_{\mathbb{T}}$ from finite $\mathbb{T}$-CW complexes to sheaves of $\mathbb{Z} / 2$-graded $\mathcal{O}_{C}$-algebras, equipped with a natural isomorphism (1.2), where the left side denotes the completion of the stalk of $E_{\mathbb{T}}(X)$ at the origin of $C$ ([11]; for a published account see $\left.[19,1]\right)$.

The bundles for which we construct trivializations $\gamma(V)$ are the virtual $\mathbb{T}$ oriented equivariant spin bundles $V$ with $c_{2}\left(V_{\mathbb{T}}\right)=0$. This requires some explanation.

Let $V$ be a $\mathbb{T}$-vector bundle over $X$. A $\mathbb{T}$-orientation $\epsilon$ on $V$ is a choice of orientation $\epsilon\left(V^{A}\right)$ on the fixed sub-bundle $V^{A}$ for each closed subgroup $A$ of $\mathbb{T}$. We say that $V$ is $\mathbb{T}$-orientable if it admits a $\mathbb{T}$-orientation; a $\mathbb{T}$ oriented vector bundle is a $\mathbb{T}$-vector bundle equipped with a $\mathbb{T}$-orientation. An isomorphism of $\mathbb{T}$-oriented vector bundles is an isomorphism of $\mathbb{T}$-vector bundles which preserves the orientations on each of the fixed sub-bundles.

A $\mathbb{T}$-equivariant spin bundle is a spin vector bundle $V$, equipped with an action of $\mathbb{T}$ on its principal spin bundle $Q \rightarrow X$. If $V$ is a $\mathbb{T}$-equivariant spin bundle with principal spin bundle $Q$, then the Borel construction $Q_{\mathbb{T}}$ is a principal spin bundle over $X_{\mathbb{T}}$; the associated spin vector bundle is $V_{\mathbb{T}}$.

Since we produce a section of the sheaf which corresponds in Grojnowski's $E_{\mathbb{T}}$ to the cohomology of the Thom spectrum of a virtual bundle, it is important to identify this sheaf precisely, rather than merely its isomorphism class. Our approach is to construct the section for a pair $V=V / X=\left(V_{0}, V_{1}\right)$ of $\mathbb{T}$-oriented equivariant spin vector bundles over $X$, representing the virtual bundle $V_{0}-V_{1}$, and then state explicitly how the construction depends on the pair. We choose not to write $V=V_{0}-V_{1}$, to emphasize that we are working with genuine vector bundles and not mere isomorphism classes. In particular the virtual bundles over $X$ do not form an abelian group. On the other hand, if $V$ is such a pair, then $X^{V}$ will denote the Thom spectrum of the virtual bundle $V_{0}-V_{1}$.

If $V=\left(V_{0}, V_{1}\right)$ is a pair of vector bundles over $X$, and $f: Y \rightarrow X$ is a map, then we write $f^{*} V$ for the pair $\left(f^{*} V_{0}, f^{*} V_{1}\right)$ of vector bundles over $Y$. A map

$$
g / f: W / Y \rightarrow V / X
$$

of pairs of vector bundles will mean a map of spaces

$$
f: Y \rightarrow X
$$


and maps of vector bundles

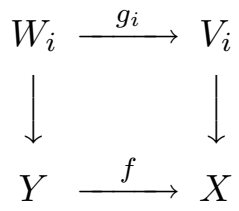

covering $f$ for $i=0,1$. Such a map will be called a pull-back if the diagram (1.4) is a pull-back for each $i$. If $V$ is a pair of $\mathbb{T}$-vector bundles, then we write $V_{\mathbb{T}}$ for the pair $\left(\left(V_{0}\right)_{\mathbb{T}},\left(V_{1}\right)_{\mathbb{T}}\right)$. If $V^{\prime}=\left(V_{0}^{\prime}, V_{1}^{\prime}\right)$ is another pair of vector bundles over $X$, then we write $V \oplus V^{\prime}$ for the pair $\left(V_{0} \oplus V_{0}^{\prime}, V_{1} \oplus V_{1}^{\prime}\right)$ over $X$.

A spin vector bundle $V$ has an integral characteristic class, twice which is the first Pontrjagin class. It is called $\frac{p_{1}}{2}$ in [7] and $\lambda$ in [8]. The isomorphism

$$
H^{4}(B S U ; \mathbb{Z}) \cong H^{4}(B \operatorname{Spin} ; \mathbb{Z})
$$

identifies it with the second Chern class, so we call it $c_{2}$ as in [2]. If $V=\left(V_{0}, V_{1}\right)$ is a pair of spin bundles, then we define $c_{2}(V)=c_{2}\left(V_{0}\right)-c_{2}\left(V_{1}\right)$.

If $V$ is a $\mathbb{T}$-equivariant vector bundle over $X$, then we write $E_{\mathbb{T}}(V)$ for the reduced equivariant elliptic cohomology of its Thom space. It is an $E_{\mathbb{T}}(X)$ module, and if $V$ is $\mathbb{T}$-orientable, then it is an invertible $E_{\mathbb{T}}(X)$-module (see section 7 and $[19,1]$ ). If $V$ and $V^{\prime}$ are two such bundles then there is a canonical isomorphism of $E_{\mathbb{T}}(X)$-modules

$$
E_{\mathbb{T}}\left(V \oplus V^{\prime}\right) \cong E_{\mathbb{T}}(V) \underset{E_{\mathbb{T}}(X)}{\otimes} E_{\mathbb{T}}\left(V^{\prime}\right) .
$$

If $V$ is a $\mathbb{T}$-oriented vector bundle, and $f: Y \rightarrow X$ is an equivariant map, then there is a canonical isomorphism

$$
E_{\mathbb{T}}\left(f^{*} V\right) \cong f^{*} E_{\mathbb{T}}(V)
$$

of sheaves of $E_{\mathbb{T}}(Y)$-modules.

If $V=\left(V_{0}, V_{1}\right)$ is a pair of $\mathbb{T}$-oriented vector bundles over $X$, then we define

$$
E_{\mathbb{T}}(V) \stackrel{\text { def }}{=} E_{\mathbb{T}}\left(V_{0}\right) \underset{E_{\mathbb{T}}(X)}{\otimes} E_{\mathbb{T}}\left(V_{1}\right)^{-1} .
$$

Equations (1.5) and (1.6) imply that the assignment $V \mapsto E_{\mathbb{T}}(V)$ is

(1) stable in the sense that if $W$ is a $\mathbb{T}$-oriented vector bundle over $X$, and if $V^{\prime}=\left(V_{0} \oplus W, V_{1} \oplus W\right)$, then there is a canonical isomorphism

$$
E_{\mathbb{T}}\left(V^{\prime}\right) \cong E_{\mathbb{T}}(V) ;
$$


(2) natural in the sense that if

$$
g / f: W / Y \rightarrow V / X
$$

is a pull-back of pairs of $\mathbb{T}$-oriented vector bundles, then there is a canonical isomorphism

$$
E_{\mathbb{T}}(g / f): E_{\mathbb{T}}(f)^{*} E_{\mathbb{T}}(V) \cong E_{\mathbb{T}}(W)
$$

of $E_{\mathbb{T}}(Y)$-algebras.

(3) exponential in the sense that if $V^{\prime}=\left(V_{0}^{\prime}, V_{1}^{\prime}\right)$ is another $\mathbb{T}$-oriented vector bundle, then there is a canonical isomorphism

$$
E_{\mathbb{T}}\left(V \oplus V^{\prime}\right) \cong E_{\mathbb{T}}(V) \underset{E_{\mathbb{T}}(X)}{\otimes} E_{\mathbb{T}}\left(V^{\prime}\right)
$$

extending (1.5)

Our main result is the following.

Theorem 1.10 (9.1) Let $V=\left(V_{0}, V_{1}\right)$ be a pair of $\mathbb{T}$-oriented equivariant spin vector bundles over a finite $\mathbb{T}-C W$ complex $X$, with the property that $c_{2}\left(V_{\mathbb{T}}\right)=0$. Then there is a canonical trivialization $\gamma(V)$ of $E_{\mathbb{T}}(V)$, whose value in $E_{\mathbb{T}}(V)^{\wedge} \cong E\left(X_{\mathbb{T}}^{V_{\mathbb{T}}}\right)$ is the Thom class provided by the (nonequivariant) sigma orientation.

The association $V \mapsto \gamma(V)$ is stable in the sense that if $W$ is a $\mathbb{T}$-oriented $\mathbb{T}$-equivariant spin bundle, and $V^{\prime}=\left(V_{0} \oplus W, V_{1} \oplus W\right)$, then

$$
\gamma\left(V^{\prime}\right)=\gamma(V)
$$

under the isomorphism (1.7). It is natural in the sense that if

$$
g / f: W / Y \rightarrow V / X
$$

is a pull-back of pairs of $\mathbb{T}$-oriented equivariant spin bundles with vanishing Borel second Chern class, then

$$
E_{\mathbb{T}}(f)^{*} \gamma(V)=\gamma\left(V^{\prime}\right)
$$

under the isomorphism (1.8). It is exponential in the sense that if $V^{\prime}=\left(V_{0}^{\prime}, V_{1}^{\prime}\right)$ is another pair of $\mathbb{T}$-oriented $\mathbb{T}$-equivariant spin bundles with $c_{2}\left(V_{\mathbb{T}}^{\prime}\right)=0$, then

$$
\gamma\left(V \oplus V^{\prime}\right)=\gamma(V) \otimes \gamma\left(V^{\prime}\right)
$$

under the isomorphism (1.9).

Remark 1.11 It is a result of Bott and Samelson ([6]; see [7] or Lemma 5.27) that a $\mathbb{T}$-equivariant spin bundle is $\mathbb{T}$-orientable. 
Remark 1.12 The class $\gamma(V)$ depends on the $\mathbb{T}$-orientation and spin structure, but otherwise requires only that $c_{2}\left(V_{\mathbb{T}}\right)=0$, i.e. that $V_{\mathbb{T}}$ admits a $B O\langle 8\rangle$-structure. We suspect that the failure of our construction to depend on the choice of $B O\langle 8\rangle$ structure on $V_{\mathbb{T}}$ reflects the fact that our elliptic curve $C$ comes as a quotient $\mathbb{C} / \Lambda$. For example in Lemma 5.20 , it is important to be able to name the point $q^{\frac{1}{n}}$. This is similar to the situation of the Ochanine genus, whose rigidity requires only that $V$ is a spin bundle.

In [1], Maria Basterra and the author showed that, under the hypotheses of the Theorem, there is a global section $\gamma(V)$ of $E_{\mathbb{T}}(V)$, whose value in the stalk at the origin of $C$ is the sigma orientation of $V_{\mathbb{T}}$; earlier Rosu [19] did the same for the orientation associated to the Euler formal group law. However, those papers do not address the trivialization, naturality, and exponential properties of the classes they construct.

The naturality is particularly hard to discern. Both of the papers $[19,1]$ closely follow [7] in their implementation of the "transfer" argument, and so all three papers depend on meticulous choices for integer representatives of the characters of the action of $\mathbb{T}$ on $\left.V\right|_{X^{\mathbb{T}}}$ and of $\mathbb{T}[n]$ on $\left.V\right|_{\mathbb{T}[n]}$ and for the orientations of $V^{\mathbb{T}}$ and $V^{\mathbb{T}}[n]$, along with surprising and not particularly intuitive results concerning the compatibility of these choices. In this paper we show that any choice will do, with no effect on the resulting Thom class, which is completely determined by the equivariant spin bundle $V$, together with the orientations of the bundles $V^{\mathbb{T}[n]}$ and $V^{\mathbb{T}}$. The argument is indifferent to the parity of $n$.

From a practical point of view there are two important new ingredients. The first is a careful account of the choice of representatives of characters (rotation numbers) for the action of $A \subseteq \mathbb{T}$ on the vector bundle $\left.V\right|_{X^{A}}$. This makes it easy to study the effect on our constructions of varying the representatives and for that matter the subgroup $A$. The second is a systematic use of the geometry of the affine Weyl group of $\operatorname{Spin}(2 d)$, and its associated theory of theta functions [16].

More important than any single practical improvement was the conceptual progress in understanding the relationship between the sigma orientation and Looijenga's work on theta functions, root systems, and elliptic curves. In order to illustrate our thinking, we state a conjecture; it is really a proposal for structure which should be expected of equivariant elliptic cohomology, once a rich enough theory has been found.

Let $E$ be the nonequivariant elliptic spectrum associated to $C$ (see (2.10) for 
a construction), and let

$$
\operatorname{sp} E\left(X_{\mathbb{T}}\right) \stackrel{\text { def }}{=} \operatorname{spf} E^{0}\left(X_{\mathbb{T}}\right) .
$$

Then $X \mapsto \operatorname{sp} E\left(X_{\mathbb{T}}\right)$ is a covariant functor from $\mathbb{T}$-spaces to formal schemes over $\operatorname{spf} E^{0}(B \mathbb{T}) \cong \widehat{C}$. To state our conjecture, it is useful to suppose that $\mathbb{T}$-equivariant elliptic cohomology is a covariant functor

$$
X \mapsto \operatorname{sp} E_{\mathbb{T}}(X)
$$

from $\mathbb{T}$-spaces to some category of (super) ringed spaces over $C$, as was proposed by Grojnowski [11] and Ginzburg-Kapranov-Vasserot [9] (and perhaps others). For example, since Grojnowski's functor $E_{\mathbb{T}}$ produces a $\mathbb{Z} / 2$-graded commutative $\mathcal{O}_{C}$-algebra, we may view $\left(C, E_{\mathbb{T}}(X)\right)$ as a ringed space sp $E_{\mathbb{T}}(X)$ over $C$. In this notation the completion isomorphism (1.2) becomes

$$
\operatorname{sp} E\left(X_{\mathbb{T}}\right) \cong\left(\operatorname{sp} E_{\mathbb{T}}(X)\right)^{\wedge} .
$$

Let $G=\operatorname{Spin}(2 d)$ with maximal torus $T$ and Weyl group $W$, and let $\check{T}=$ $\operatorname{hom}(\mathbb{T}, T)$ be the lattice of cocharacters. The formal scheme $\breve{T} \otimes \widehat{C}$ carries a natural action of $W$, and the splitting principle gives an isomorphism (see section 4)

$$
B G_{E} \cong(\check{T} \otimes \widehat{C}) / W
$$

Suppose that $V$ is a $\mathbb{T}$-vector bundle over $X$, and that $V_{\mathbb{T}}$ has structure group $G$. The map

$$
X \rightarrow B G
$$

classifying $V$ induces in $E$-cohomology a map

$$
\operatorname{sp} E\left(X_{\mathbb{T}}\right) \rightarrow(\check{T} \otimes \widehat{C}) / W .
$$

Looijenga constructs a holomorphic line bundle $\mathcal{A}$ over $(\check{T} \otimes C) / W([16]$; see also section 5.2). The sigma function $\sigma$ defines a global holomorphic section $\sigma_{d}$ of $\mathcal{A}$, whose zeroes define an ideal sheaf $\mathcal{I}$ on $(\check{T} \otimes C) / W$, such that $\sigma_{d}$ is a trivialization of $\mathcal{A} \otimes \mathcal{I}$. Our conjecture is the following; it is stated with a little more detail as Conjecture 10.1.

Conjecture 1.14 The $\mathbb{T}$-equivariant elliptic cohomology $E_{\mathbb{T}}$ associated to the elliptic curve $C$ should associate to the $\mathbb{T}$-equivariant spin bundle $V / X$ a map

$$
f: \operatorname{sp} E_{\mathbb{T}}(X) \rightarrow(\check{T} \otimes C) / W,
$$

which upon completion gives the map (1.13). Writing $\mathcal{I}(V)=f^{*} \mathcal{I}$ and $\mathcal{A}(V)=$ $f^{*} \mathcal{A}$, this map should have the following properties. 
(1) There is a canonical isomorphism

$$
\mathcal{I}(V) \cong E_{\mathbb{T}}(V)
$$

of line bundles over $\operatorname{sp} E_{\mathbb{T}}(X)$.

(2) Suppose that $V^{\prime}$ is another $\mathbb{T}$-equivariant spin bundle. If

$$
c_{2}\left(V_{\mathbb{T}}\right)=c_{2}\left(V_{\mathbb{T}}^{\prime}\right),
$$

then $\mathcal{A}(V) \cong \mathcal{A}\left(V^{\prime}\right)$; indeed a $\mathbb{T}-B O\langle 8\rangle$ structure on $V-V^{\prime}$ determines a trivialization of $\mathcal{A}(V) \otimes \mathcal{A}\left(V^{\prime}\right)^{-1}$.

In the notation of the Theorem, let $\sigma(V)=f^{*}\left(\sigma_{d}\right)$. If $V-V^{\prime}$ is a $\mathbb{T}-B O\langle 8\rangle$ bundle, then $\sigma(V) / \sigma\left(V^{\prime}\right)$ is a trivialization of

$$
\frac{\mathcal{A}(V) \otimes \mathcal{I}(V)}{\mathcal{A}\left(V^{\prime}\right) \otimes \mathcal{I}\left(V^{\prime}\right)} \cong \frac{E_{\mathbb{T}}(V)}{E_{\mathbb{T}}\left(V^{\prime}\right)}
$$

this is the equivariant sigma orientation for $V-V^{\prime}$. From this point of view, the sigma function always determines a trivialization of the line bundle $\mathcal{A}(V) \otimes$ $\mathcal{I}(V)$; when $c_{2}(V)=0, \mathcal{A}(V)$ is trivial and so this gives a Thom class. The letter $\mathcal{A}$ stands for "anomaly".

Early versions of this paper were attempts to prove the Conjecture 1.14 for Grojnowski's $E_{\mathbb{T}}$, and then to deduce Theorem 1.10 from it. However, for reasons we explain in section 10, we were only occasionally able to convince even ourselves of those proofs. Eventually, detailed consideration of the consequences of the Conjecture led us to the formulae in section 5.4 and so to concrete proofs.

In section 10 we do construct the map in the Conjecture for a functor which captures the behavior of the stalks of Grojnowski's functor. The argument uses the same information we used in section 5.4 and section 8 to produce the equivariant Thom class. The functor we study in section 10 was also inspired by Greenlees's rational $\mathbb{T}$-equivariant elliptic spectra [10] and by Hopkins's work on characters in elliptic cohomology [12]. Indeed we hope that Greenlees's rational $\mathbb{T}$-equivariant elliptic spectra will admit a proof of the conjecture, and so give an account of the rational circle-equivariant sigma orientation.

The rest of the paper proceeds as follows. In section 2 we summarize some of the notation which recurs throughout the paper. We discuss complex-orientable cohomology theories in general and ordinary and elliptic cohomology theories in particular. In section 3 we state in a useful form some standard facts about $\mathbb{T}$-equivariant principal $G$-bundles.

In section 4 we interpret the analysis of section 3 in the presence of a (rational) complex-orientable cohomology theory. We begin section 5 with an interlude 
(section 5.1) on degree-four characteristic classes. In section 5.2 we recall a result essentially due to [16], that a degree-four characteristic class $\xi \in H^{4}(B G ; \mathbb{Z})$ gives rise to a $W$-equivariant line bundle $\mathcal{L}(\xi)$ over $(\check{T} \otimes C)$. We define a theta function of level $\xi$ for $G$ to be a $W$-invariant holomorphic section of the line bundle $\mathcal{L}(\xi)$; by the splitting principle, the Taylor series expansion of such a theta function defines a characteristic class of principal $G$-bundles. The sigma function provides the most important examples for us, and so we discuss it in section 5.3.

Section 5.4 is the heart of the paper. In it we use the results of section $3-5.3$ to construct some holomorphic characteristic classes for $\mathbb{T}$-equivariant principal $G$-bundles which are the building blocks of the Thom classes in section 8 and section 9 .

In section 6 we recall the construction of Grojnowski's analytic $\mathbb{T}$-equivariant elliptic cohomology associated to a lattice $\Lambda \subset \mathbb{C}$. In section 7 we review the equivariant elliptic cohomology of Thom complexes $[19,1]$, recalling what is involved in constructing a global section of $E_{\mathbb{T}}(V)$, where $V$ is (virtual) $\mathbb{T}$ oriented vector bundle.

In section 9 we construct the equivariant sigma orientation, proving Theorem 1.10. In section 8 we prove the following related result. Let $G$ be a spinor group, and let $G^{\prime}$ be a simple and simply connected compact Lie group. Let $V$ be a $\mathbb{T}$-equivariant $G$-bundle over a finite $\mathbb{T}$-CW complex $X$, and let $V^{\prime}$ be a $\mathbb{T}$-equivariant $G^{\prime}$-bundle. Let

$$
\Sigma\left(V_{\mathbb{T}}\right) \in E\left(X_{\mathbb{T}}^{V_{\mathbb{T}}}\right)
$$

be the Thom class given by the Weierstrass sigma function (see Definition 4.13). Suppose that $\xi^{\prime}$ is a degree-four characteristic classes for $G^{\prime}$, with the property that

$$
c_{2}\left(V_{\mathbb{T}}\right)=\xi^{\prime}\left(V_{\mathbb{T}}^{\prime}\right) .
$$

Suppose that $\theta^{\prime}$ is a theta function for $G^{\prime}$ of level $\xi^{\prime}$.

Theorem 1.15 (8.6) A $\mathbb{T}$-orientation $\epsilon$ on $V$ determines a canonical global section $\gamma=\gamma\left(V, V^{\prime}, \epsilon\right)$ of $E_{\mathbb{T}}(V)^{-1}$, whose value in $E_{\mathbb{T}}(V)_{0}^{-1}$ is $\theta^{\prime}\left(V_{\mathbb{T}}^{\prime}\right) \Sigma\left(V_{\mathbb{T}}\right)^{-1}$. The formation of $\gamma$ is natural in the sense if

$$
\begin{aligned}
g / f: W / Y & \rightarrow V / X \\
g^{\prime} / f: W^{\prime} / Y & \rightarrow V^{\prime} / X
\end{aligned}
$$

are pull-backs of vector bundles, then under the isomorphism

$$
E_{\mathbb{T}}(g / f): E_{\mathbb{T}}(f)^{*} E_{\mathbb{T}}(V) \cong E_{\mathbb{T}}(W),
$$


we have

$$
E_{\mathbb{T}}(f)^{*} \gamma\left(V, V^{\prime}, \epsilon\right)=\gamma\left(W, W^{\prime}, g^{*} \epsilon\right) .
$$

In particular, suppose that $V^{\prime}$ is an equivariant $\operatorname{Spin}\left(2 d^{\prime}\right)$-vector bundle over $X$, and $V$ is an equivariant $\operatorname{Spin}(2 d)$ vector bundle. Suppose that $\theta^{\prime}$ is the character of a representation of $L \operatorname{Spin}\left(2 d^{\prime}\right)$ of level $k$ : then it is a theta function of level $k c_{2}$ for $\operatorname{Spin}\left(2 d^{\prime}\right)$. If

$$
c_{2}\left(V_{\mathbb{T}}\right)=k c_{2}\left(V_{\mathbb{T}}^{\prime}\right),
$$

then Theorem 1.15 gives a global section $\gamma$ of $E_{\mathbb{T}}(V)^{-1}$. If $X$ is a manifold and $V$ is the tangent bundle of $X$, then the Pontrjagin-Thom construction for the map $\pi: X \rightarrow *$ gives a map

$$
\left(E_{\mathbb{T}}(\pi)\right)_{*} E_{\mathbb{T}}(V)^{-1} \rightarrow E_{\mathbb{T}}(*)=\mathcal{O}_{C}
$$

of $\mathcal{O}_{C}$-modules which takes $\gamma$ to the equivariant Witten genus of $V$ twisted by the characteristic class $\theta^{\prime}\left(V_{\mathbb{T}}^{\prime}\right)$. Since the global sections of $\mathcal{O}_{C}$ are the constants, we have the following result of Kefeng Liu [15].

Corollary 1.16 Under these conditions, the equivariant Witten genus of $X$ twisted by $\theta\left(V_{\mathbb{T}}^{\prime}\right)$ is constant.

Remark 1.17 Liu states a condition on $p_{1}$ instead of $c_{2}$.

If section 5.4 is the heart of the paper, then section 10 is the soul. There we discuss Conjecture 1.14, the study of which led to the results we report here. We give a refinement of the conjecture (Conjecture 10.1), and we explain how the arguments in this paper support it. We show how to construct the map of Conjecture 1.14 for a functor which captures the behavior of the stalks of Grojnowski's functor; the construction is essentially a "transfer formula" in the sense of [7]. We show that the nonequivariant version of the conjecture is true, and sheds light on the nonequivariant sigma orientation. Because the characters of representations of the loop group $L G$ are sections of the line bundle $\mathcal{A}$, it illuminates the relationship between the sigma orientation, equivariant elliptic cohomology, and representations of loop groups $[5,3]$.

\section{$1.1 \quad$ Acknowledgments}

My first debt is to Maria Basterra. Our work on [1] led directly to the results in this paper, and I have very much enjoyed and benefitted from our collaboration. 
Maria was to have been an author of this paper as well, and I have reluctantly accepted her request to withdraw her name from it. I thank Alejandro Adem for inviting me to visit Madison; it was during the eight-hour round-trip drive that the the function $F$ (5.16) was discovered, along with its properties as described in section 5.4. I thank John Greenlees, Haynes Miller, Jack Morava, and Charles Rezk for useful conversations. I thank Haynes Miller, Jack Morava, and Amnon Neeman for encouraging me to record the ideas in section 10; I hope they do not regret the result. My work on elliptic cohomology has been profoundly influenced by Mike Hopkins, and I am grateful to him for his work on the subject and his generosity to me in particular.

The author is supported by NSF grant DMS-0071482. This paper was completed during a visit to the Newton Institute for Mathematical Sciences, during which time the author was supported by the Center for Advanced Study of the University of Illinois at Urbana-Champaign.

\section{Notation}

\subsection{Abelian groups}

Let $\mathcal{C}$ be a category with finite products. The category $A \mathcal{C}$ of abelian groups in $\mathcal{C}$ is an additive category. In fact $A \mathcal{C}$ is tensored over the category of finitely generated free abelian groups. That is, a finitely generated free abelian group $F$ and an abelian group $X$ of $\mathcal{C}$ determine (naturally in $F$ and $X$ ) an object $F \otimes X$ of $A \mathcal{C}$, with a natural isomorphism

$$
A \mathcal{C}[F \otimes X, Y] \cong \text { (abelian groups) }[F, A \mathcal{C}[X, Y]] .
$$

If $A$ is an abelian group written additively, and $M$ is an abelian group written multiplicatively, then we write $m^{a}$ for the element $a \otimes m$ of $A \otimes M$. Similarly, if $M^{\prime}$ is an abelian group, $u \in M^{\prime}$, and $m \in \operatorname{hom}\left(M^{\prime}, M\right)$ then we may write $u^{m}$ for $m(u)$.

We write $\mathbb{G}_{a}$ for the additive group, and $\mathbb{G}_{m}$ for the multiplicative group.

\subsection{Lie groups and the group $\check{T} \otimes X$}

In general, the letter $G$ will stand for a compact Lie group with maximal torus $T$ with Weyl group $W$. We define

$$
\begin{aligned}
& \check{T} \stackrel{\text { def }}{=} \operatorname{hom}[\mathbb{T}, T] \\
& \hat{T} \stackrel{\text { def }}{=} \operatorname{hom}[T, \mathbb{T}]
\end{aligned}
$$


to be the lattices of cocharacters and characters. We write

$$
c: G \rightarrow \operatorname{Aut}(G)
$$

for the action of $G$ on itself by conjugation:

$$
c_{g} h=g h g^{-1} .
$$

If $X$ is an abelian group in any category, then the tensor product $\check{T} \otimes X$ carries an action of the Weyl group $W$. If $r$ is the rank of $G$, then $\check{T} \otimes X$ is isomorphic to $X^{r}$.

\section{$2.3 \quad$ Elliptic curves}

Fix $\tau$ in the complex upper half plane, and let $\Lambda$ be the lattice

$$
\Lambda=2 \pi i \mathbb{Z}+2 \pi i \tau \mathbb{Z} .
$$

The complex numbers appear in this paper both as a ring and as an analytic variety. To avoid confusion we write $\mathbb{A}_{\text {an }}^{1}$ for the complex numbers regarded as an analytic variety. Similarly, we write $\mathbb{G}_{a}^{\text {an }}$ for $\mathbb{A}_{\text {an }}^{1}$ with its additive structure of abelian topological group. We write $z$ for the standard coordinate on $\mathbb{G}_{a}$ and also on $\mathbb{A}^{1}, \mathbb{A}_{\text {an }}^{1}, \mathbb{G}_{a}^{\text {an }}$, etc. We write $\mathbb{G}_{m}^{\text {an }}$ for $\left(\mathbb{A}_{\text {an }}^{1}\right)^{\times}$with its multiplicative group structure. We set

$$
\begin{aligned}
u^{r} & =e^{r z} \\
q^{r} & =e^{2 \pi i r \tau}
\end{aligned}
$$

for $r \in \mathbb{Q}$, and we let $C$ be the elliptic curve

$$
C=\mathbb{C} / \Lambda=\mathbb{G}_{a}^{\text {an }} / \Lambda \cong \mathbb{C}^{\times} / q^{\mathbb{Z}}=\mathbb{G}_{m}^{\text {an }} / q^{\mathbb{Z}} .
$$

We write $\wp$ for the covering map

$$
\mathbb{A}_{\text {an }}^{1} \stackrel{\wp}{\rightarrow} C .
$$

If $V$ is an open set in a complex analytic variety, then we write $\mathcal{O}_{V}$ for the sheaf of holomorphic functions on $V$.

If $A$ is an abelian topological group and $a \in A$, when we write $\tau_{a}$ for the translation map; and if $V \subset A$ is an open set, then we write

$$
V-g \stackrel{\text { def }}{=} \tau_{-g}(V) .
$$

Definition 2.2 An open set $U$ of $C$ is small if it is connected and $\wp^{-1} U$ is a union of connected components $V$ with the property that

$$
\left.\wp\right|_{V}: V \rightarrow U
$$

is an isomorphism. 
If $U$ is small and $V$ is a component of $\wp^{-1} U$, then the covering map induces an isomorphism

$$
\mathcal{O}_{U} \cong \mathcal{O}_{V}
$$

In particular, if $U$ contains the origin of $C$, then there is a unique component $V$ of $\wp^{-1} U$ containing 0 . This determines a $\mathbb{C}[z]$-algebra structure on $\mathcal{O}_{U}$, and a $\mathbb{C}\left[z, z^{-1}\right]$ structure on $\left.\mathcal{O}_{U}\right|_{U \backslash 0}$.

\section{$2.4 \quad$ Ringed spaces}

Grojnowski's $\mathbb{T}$-equivariant elliptic cohomology is a contravariant functor

$$
E_{\mathbb{T}}:(\text { finite } \mathbb{T} \text {-CW complexes }) \rightarrow\left(\mathbb{Z} / 2 \text {-graded } \mathcal{O}_{C} \text {-algebras }\right)
$$

(see section 6 and $[11,19,1]$ ). At roughly the same time as [11], GinzburgKapranov-Vasserot [9] proposed that the $\mathbb{T}$-equivariant elliptic cohomology associated to an elliptic curve $C$ should be a covariant functor

$$
E_{\text {ideal }}:(\mathbb{T} \text {-spaces }) \rightarrow(\text { schemes }) / C,
$$

although they gave no independent construction of such a functor.

These are meant to be related by the formula

$$
E_{\mathbb{T}}(X)=f_{*} \mathcal{O}_{E_{\text {ideal }}(X)},
$$

where

$$
f: E_{\text {ideal }}(X) \rightarrow C
$$

is the structural map. Grojnowksi's functor can not quite be of the form (2.4), since in $(2.3) \mathcal{O}_{C}$ is the sheaf of holomorphic functions on the analytic space $C=\mathbb{G}_{a}^{\text {an }} / \Lambda$. However, it does give a covariant functor

$$
\text { (finite } \mathbb{T} \text {-spaces }) \rightarrow(\text { ringed spaces }) / C \text {. }
$$

Precisely, we have the following.

Definition 2.5 By a (super, or $\mathbb{Z} / 2$-graded) ringed space we shall mean a pair $\left(X, \mathcal{O}_{X}\right)$ consisting of a space $X$ and a sheaf $\mathcal{O}_{X}$ of $\mathbb{Z} / 2$-graded rings on $X$. A map of ringed spaces

$$
f=\left(f_{1}, f_{2}\right):\left(X, \mathcal{O}_{X}\right) \rightarrow\left(Y, \mathcal{O}_{Y}\right)
$$

consists of a map of spaces $f_{1}: X \rightarrow Y$ and a map of sheaves of $\mathbb{Z} / 2$-graded commutative algebras over $Y$

$$
f_{2}: \mathcal{O}_{Y} \rightarrow\left(f_{1}\right)_{*} \mathcal{O}_{X}
$$


The resulting category of ringed spaces will be denoted $\mathcal{R}$. If $\mathcal{X}=\left(X, \mathcal{O}_{X}\right)$ is a ringed space and $U$ is an open set of $X$, then we may write $\mathcal{X}(U)$ in place of $\mathcal{O}_{X}(U)$

If $X$ is a finite $\mathbb{T}$-CW complex, then

$$
\operatorname{sp} E_{\mathbb{T}}(X) \stackrel{\text { def }}{=}\left(C, E_{\mathbb{T}}(X)\right)
$$

is a ringed space. If

$$
f: Y \rightarrow X
$$

is a map of finite $\mathbb{T}$-CW complexes then we define

$$
\operatorname{sp} E_{\mathbb{T}}(f): \operatorname{sp} E_{\mathbb{T}}(Y) \rightarrow \operatorname{sp} E_{\mathbb{T}}(X)
$$

to be the identity on $C$ and

$$
E_{\mathbb{T}}(f): E_{\mathbb{T}}(X) \rightarrow E_{\mathbb{T}}(Y)
$$

on the structure sheaves. In this we obtain a covariant functor

$$
\operatorname{sp} E_{\mathbb{T}}: \text { (finite } \mathbb{T} \text {-CW complexes) } \rightarrow \mathcal{R} / C .
$$

We have found this point of view to be extremely helpful, and so we have adopted it in writing this paper.

\subsection{Ordinary cohomology}

If $R$ is a commutative ring, let $H R$ denote ordinary cohomology with coefficients in $R$. If $X$ is a space, then we write

$$
\operatorname{sp} H R(X) \stackrel{\text { def }}{=} \operatorname{spec}\left(H R^{\text {even }}(X)\right)
$$

for the scheme over spec $R$ associated to the cohomology of $X$. We may also view $\operatorname{sp} H R(X)$ as the ringed space with underlying space $\operatorname{spec}\left(H R^{\text {even }}(X)\right)$ and structure sheaf associated to

$$
H R^{\text {even }}(X) \oplus H R^{\text {odd }}(X) .
$$

We shall write $H$ for $H \mathbb{C}$, cohomology with complex coefficients. 


\subsection{Equivariant cohomology}

If $X$ is a space with a circle action, sp $H R\left(X_{\mathbb{T}}\right)$ is a scheme over sp $H R(B \mathbb{T})$, which we denote sp $H R(X)$. We choose a generator of the character group of $\mathbb{T}$, and write $z$ for the resulting generator of $H \mathbb{Z}^{2}(B \mathbb{T})$; this gives an isomorphism

$$
\operatorname{sp} H R(B \mathbb{T}) \cong\left(\mathbb{G}_{a}\right)_{R}
$$

of group schemes over $\operatorname{spec} R$. We shall use (2.6) to view $\operatorname{sp} H R\left(X_{\mathbb{T}}\right)$ as a scheme over $\mathbb{A}_{R}^{1}$.

We recall [18] that equivariant cohomology satisfies a localization theorem.

Theorem 2.7 If $X$ has the homotopy type of a finite $\mathbb{T}$-CW complex (e.g. if $X$ is a compact $\mathbb{T}$-manifold), then the natural map

$$
\operatorname{sp} H\left(X_{\mathbb{T}}^{\mathbb{T}}\right) \rightarrow \operatorname{sp} H\left(X_{\mathbb{T}}\right)
$$

induces an isomorphism over $\operatorname{spec} \mathbb{C}\left[z, z^{-1}\right] \subset \operatorname{sp} H(B \mathbb{T})$.

\section{Holomorphic cohomology}

Let $\mathbb{A}_{\text {an }}^{1}$ be the analytic complex plane, so $\mathcal{O}_{\mathbb{A}_{a n}^{1}}$ is the sheaf of holomorphic functions on $\mathbb{C}$. Because of the natural maps

$$
\mathbb{A}_{\text {an }}^{1} \rightarrow \mathbb{A}_{\mathbb{C}}^{1} \rightarrow \mathbb{A}^{1} \cong \operatorname{sp} H \mathbb{Z}(B \mathbb{T})
$$

we may view $z$ as a function on $\mathbb{A}_{\text {an }}^{1}$. Given a $\mathbb{T}$-space $X$ we define the holomorphic cohomology of $X$ to be the sheaf of super $\mathcal{O}_{\mathbb{A}_{\mathrm{an}}^{1}}$-algebras given by

$$
\mathcal{H}(X ; U) \stackrel{\text { def }}{=} H \mathbb{C}\left(X_{\mathbb{T}}\right) \underset{\mathbb{C}[z]}{\otimes} \mathcal{O}_{\mathbb{A}_{\text {an }}^{1}}(U) .
$$

We view $\mathcal{H}(X)$ as the structure sheaf of a ringed space $(2.5)$ sp $\mathcal{H}(X)$ over $\mathbb{A}_{\text {an }}^{1}$, namely the the pull-back in the diagram

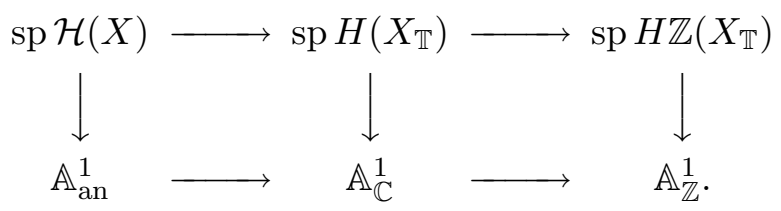




\section{The stalk of holomorphic cohomology}

Let

$$
\mathbb{A}_{\mathrm{an}, 0}^{1}=\operatorname{spec}\left(\mathcal{O}_{\mathbb{A}_{\mathrm{an}}^{1}, 0}\right)
$$

be the local scheme associated to the stalk of $\mathcal{O}_{\mathbb{A}_{\text {an }}^{1}}$ at the origin. The stalk of $\mathcal{H}(X)$ at the origin is

$$
\mathcal{H}(X)_{0} \cong H\left(X_{\mathbb{T}}\right) \underset{\mathbb{C}[z]}{\otimes}\left(\mathcal{O}_{\mathbb{A}_{\text {an }}^{1}, 0}\right) .
$$

We write $\operatorname{sp} H\left(X_{\mathbb{T}}\right)_{0}$ for the resulting scheme over $\mathbb{A}_{\mathrm{an}, 0}^{1}$.

\section{Periodic Borel cohomology}

Let $H P$ denote periodic ordinary cohomology with complex coefficients: that is,

$$
H P=\bigvee_{k \in \mathbb{Z}} \Sigma^{2 k} H
$$

so

$$
\pi_{*} H P=\mathbb{C}\left[v, v^{-1}\right]
$$

with $v \in \pi_{2} H P$. Then

$$
H P^{0}(B \mathbb{T}) \cong \mathbb{C} \llbracket z \rrbracket
$$

so spf $H P^{0}(B \mathbb{T})=\left(\mathbb{A}_{\text {an }}^{1}\right)_{0}^{\wedge}=\left(\mathbb{A}_{\mathbb{C}}^{1}\right)_{0}^{\wedge}$, and $H P^{0}\left(X_{\mathbb{T}}\right)$ is the ring of formal functions on the pull-back sp $H P\left(X_{\mathbb{T}}\right)$ in the diagram of formal schemes

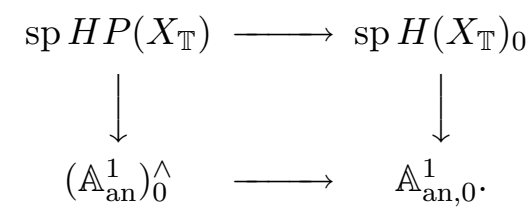

Summarizing, we have a collection of forms of ordinary cohomology

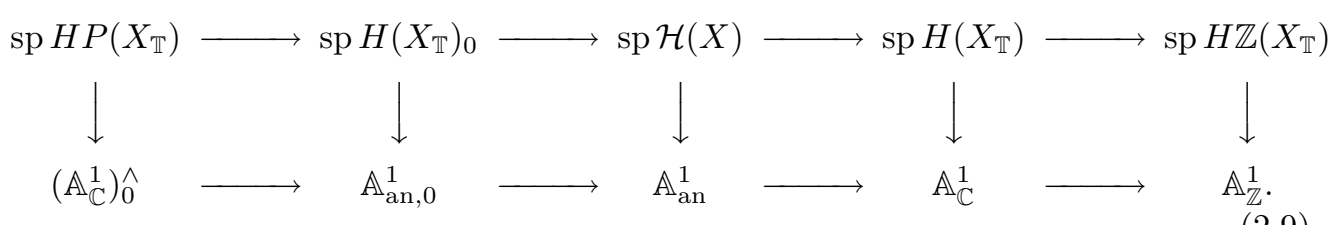




\subsection{Generalized cohomology}

\section{Even periodic ring spectra}

A ring spectrum $E$ will be called "even periodic" if $\pi_{\text {odd }} E=0$ and $\pi_{2} E$ contains a unit of $\pi_{*} E$.

If $E$ is an even periodic ring spectrum, and if $X$ is a space, then we shall write $E^{*} X$ for the unreduced cohomology of $X$. As in [2], we write $\operatorname{sp} E(X)$ for the formal scheme

$$
\operatorname{sp} E(X)=\operatorname{colim}_{F \subset X} \operatorname{spec} E^{0} F
$$

over $S_{E}=\operatorname{spec} E^{0}(*)$; the colimit is over the compact subsets of $X$.

An even periodic ring spectrum is always complex-orientable. In particular

$$
P_{E} \stackrel{\text { def }}{=} \operatorname{sp} E(B \mathbb{T})
$$

is a (commutative, one-dimensional) formal group over $S_{E}$. For example, let $H P \mathbb{Z}$ denote periodic ordinary cohomology with integer coefficients. Then $P_{H P \mathbb{Z}}=\widehat{\mathbb{G}}_{a}$.

\section{Borel cohomology}

If $E$ is an even periodic ring spectrum, and $X$ is a space with a circle action, then the projection

$$
X_{\mathbb{T}} \rightarrow B \mathbb{T}
$$

induces a map

$$
\operatorname{sp} E\left(X_{\mathbb{T}}\right) \rightarrow P_{E},
$$

making

$$
\operatorname{sp} E\left(X_{\mathbb{T}}\right)=\operatorname{spf} E^{0}\left(X_{\mathbb{T}}\right)
$$

into a formal scheme over the formal group of $E$.

\section{Elliptic spectra}

We recall [2] that an elliptic spectrum is a triple $(E, C, t)$ consisting of

(1) an even periodic ring spectrum $E$,

(2) a (generalized) elliptic curve $C$ over $S_{E}$, and

(3) an isomorphism of formal groups

$$
t: \widehat{C} \cong P_{E} .
$$




\section{Rational elliptic spectra}

Let

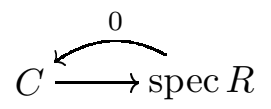

be an elliptic curve with identity 0 over a $\mathbb{Q}$-algebra $R$, and let $\underline{\omega}=0^{*} \Omega_{C / R}^{1}$. If $\omega$ is a trivialization of $\underline{\omega}$, then there is a canonical isomorphism of formal groups over $R$

$$
\widehat{C} \stackrel{\log _{\omega}}{\cong} \widehat{\mathbb{G}}_{a}
$$

with the property that $0^{*} \log _{\omega}^{*} d z=0^{*} d z$.

Let $\Gamma^{\times}(\underline{\omega})$ be the functor from rings to sets which given by

$$
\Gamma^{\times}(\underline{\omega})(T)=\left\{(j, \omega) \mid j: \operatorname{spec} T \rightarrow \operatorname{spec} R, \omega \text { a trivializaton of } j^{*} \underline{\omega}\right\} .
$$

If $\omega$ is a trivialization of $\underline{\omega}$, then the trivializations of $j^{*} \underline{\omega}$ are of the form $u j^{*} \omega$ for $u \in T^{\times}$, so

$$
\Gamma^{\times}(\underline{\omega}) \cong \operatorname{spec} R\left[u, u^{-1}\right]
$$

Let $S=\mathcal{O}\left(\Gamma^{\times}(\underline{\omega})\right)$.

The logarithm then gives a canonical isomorphism of formal groups

$$
\widehat{C}_{S} \stackrel{\log _{\widehat{C}}}{\longrightarrow}\left(\widehat{\mathbb{G}}_{a}\right)_{S}
$$

by the formula

$$
(c, \omega) \mapsto\left(\log _{\omega} c, \omega\right) .
$$

If $H S\left[v, v^{-1}\right]$ is the even periodic ring spectrum such that

$$
\left(H S\left[v, v^{-1}\right]\right)^{*} X \stackrel{\text { def }}{=} H^{*}\left(X ; S\left[v, v^{-1}\right]\right),
$$

where we take $v$ to have degree 2 and $u \in S$ to have degree zero, then

$$
P_{H S\left[v, v^{-1}\right]}=\left(\widehat{\mathbb{G}}_{a}\right)_{S},
$$

and we have an elliptic spectrum

$$
\left(H S\left[v, v^{-1}\right], C, \log _{\widehat{C}}\right) .
$$

Alternatively, given over $R$ a trivialization $\omega$ of $\underline{\omega}$ we have the elliptic spectrum $\left(H R\left[v, v^{-1}\right], C, \log _{\omega}\right)$. 


\section{Complex elliptic spectra}

Recall (2.8) that $H P$ denotes periodic ordinary cohomology with complex coefficients.

The projection $\wp: \mathbb{G}_{a}^{\text {an }} \rightarrow C$ induces an isomorphism of formal groups

$$
\widehat{\wp}: \widehat{\mathbb{G}}_{a} \rightarrow \widehat{C} \text {. }
$$

There is a unique cotangent vector $\omega$ such that

$$
\wp^{*} \omega=0^{*} d z \text {. }
$$

We have

$$
\log _{\omega}=(\widehat{\wp})^{-1}
$$

and so an elliptic spectrum

$$
\left(H P, C, \widehat{\wp}^{-1}\right)=\left(H P, C, \log _{\omega}\right) .
$$

\section{Principal bundles with an action of the circle}

\subsection{A-bundles over trivial $A$-spaces}

Let $A$ be a closed subgroup of the circle $\mathbb{T}$. Suppose that $G$ is a connected compact Lie group with maximal torus $T$, and let $\pi: Q \rightarrow Y$ be a principal $G$ bundle over a connected space $Y$. Suppose that $A$ acts on $Q / Y$, fixing $Y$. The group of automorphisms of $Q / Y$ is the group of sections $\Gamma\left(\left(Q \times{ }_{G} G^{c}\right) / Y\right)$, and an action of $A$ on $Q / Y$ is equivalent to a section $a \in \Gamma\left(\left(Q \times{ }_{G} \operatorname{hom}\left[A, G^{c}\right]\right) / Y\right)$ (The notation $G^{c}$ refers to $G$ with $G$ acting on it by conjugation).

Since $G$ is connected and $A$ is (topologically) cyclic, every $G$-orbit in hom $[A, G]$ intersects $\operatorname{hom}[A, T]$ nontrivially: that is, the map

$$
\operatorname{hom}[A, T] \rightarrow \operatorname{hom}\left[A, G^{c}\right] \rightarrow \operatorname{hom}\left[A, G^{c}\right] / G
$$

is surjective.

In particular hom $\left[A, G^{c}\right] / G$ is discrete. Its points label the connected components of $Q \times{ }_{G} \operatorname{hom}\left[A, G^{c}\right]$ via the surjective map

$$
Q \times{ }_{G} \operatorname{hom}\left[A, G^{c}\right] \rightarrow \operatorname{hom}[A, G] / G .
$$

Since $Y$ is connected, we may choose a homomorphism $m \in \operatorname{hom}[A, T]$ such that, for all $x \in Y$, there is a $p \in Q$ such that

$$
a(x)=[p, m] ;
$$


the square brackets indicate the class in the Borel construction of the element $(p, m) \in Q \times \operatorname{hom}[A, G]$. The choice of $m$ determines $p$ only up to the centralizer $Z(m)$ in $G$ of the homomorphism $m$.

Definition 3.1 A reduction of the action of $A$ on $Q$ is a homomorphism

$$
m: A \rightarrow T
$$

such that, for all $x \in Y$, there is a $p \in Q$ such that

$$
a(x)=[p, m] .
$$

The terminology is justified by the following observation. Let

$$
Q(m)=\{p \in Q \mid[p, m]=a(\pi(p))\} .
$$

Then $\left.\pi\right|_{Q(m)}: Q(m) \rightarrow Y$ is a principal $Z(m)$ bundle over $Y$, the reduction of the structure group of $Q$ to $Z(m)$. In other words, we have given a factorization

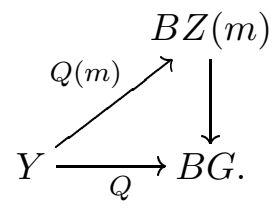

Let

$$
W(m)=\{w \in W \mid w m=m\}
$$

be the stabilizer of $m$. One sees that $T$ is a maximal torus of $Z(m)$, with Weyl group $W(m)$.

Example 3.3 Suppose that $G=U(n)$ is the unitary group with its maximal torus $T=\Delta\left(z_{1}, \ldots, z_{n}\right)$ of diagonal matrices, and suppose that $A=\mathbb{T}$. Every homomorphism

$$
m: \mathbb{T} \rightarrow T
$$

is conjugate in $U(n)$ to one of the form

$$
m(z)=\Delta\left(z^{m_{1}}, \ldots, z^{m_{1}}, z^{m_{2}}, \ldots, z^{m_{2}}, \ldots, z^{m_{r}}, \ldots, z^{m_{r}}\right),
$$

where $m_{i} \in \mathbb{Z}$. Let $d_{j}$ be the multiplicity of $m_{j}$; then the centralizer is the block-diagonal matrix

$$
Z(m)=\left[\begin{array}{lll}
U\left(d_{1}\right) & & \\
& \cdots & \\
& & U\left(d_{r}\right)
\end{array}\right],
$$


with Weyl group

$$
W(m)=\Sigma_{d_{1}} \times \cdots \times \Sigma_{d_{r}} \subset \Sigma_{n}=W .
$$

If $V$ is a $\mathbb{T}$-equivariant complex vector bundle over a connected trivial $\mathbb{T}$-space $Y$, and if this $m$ is a reduction of the action of $\mathbb{T}$ on the principal bundle of $V$, then the reduction of the structure group to $Z(m)$ corresponds to the decomposition of $V$ as the direct sum

$$
V \cong V\left(m_{1}\right) \oplus \cdots \oplus V\left(m_{r}\right),
$$

where $\mathbb{T}$ acts on the fiber of $V\left(m_{j}\right)$ by the character $z^{m_{j}}$.

The composition

$$
g(m): A \times Z(m) \stackrel{m \times Z(m)}{\longrightarrow} T \times Z(m) \rightarrow Z(m)
$$

is a group homomorphism, and so we have a map

$$
B A \times B Z(m) \stackrel{B g(m)}{\longrightarrow} B Z(m) .
$$

The following Lemma will be used directly to prove Lemma 5.5, a calculation of degree-four characteristic classes. Moreover, the algebro-geometric form of this diagram after applying a complex-orientable cohomology theory (see Lemma 4.2) captures the essential point of the "transfer argument" of [7], as we explain in Remark 10.7.

Lemma $3.4 \quad$ (1) The diagram

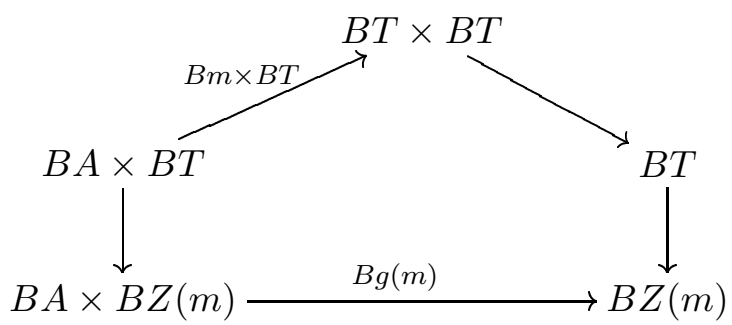

commutes.

(2) The map $B g(m)$ classifies the principal $Z(m)$-bundle $E Z(m)_{A}=E A \times_{A}$ $E Z(m)$ over $B A \times B Z(m)$.

Proof The first part is easy. For the second part, it suffices to construct a map of principal $Z(m)$-bundles over $B A \times B Z(m)$. The map

$$
E g(m): E A \times E Z(m) \rightarrow E Z(m)
$$


factors through $E A \times{ }_{A} E Z(m)$, and gives the desired map. (Note that the map $E g(m)$ is obtained by constructing $E A$ and $E Z(m)$ functorially as spaces with actions on the same side, say the left. In forming $E A \times_{A} E Z(m)$, one makes $A$ act on the right of $E A$ by the inverse.)

If $m^{\prime}: A \rightarrow T$ is another reduction of the action of $A$ on $Q$, then $m$ and $m^{\prime}$ differ by conjugation in $G$, and we have for some $g$ in $G$ a commutative diagram

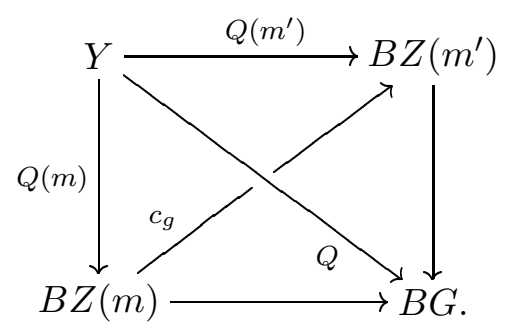

\subsection{The case of a connected centralizer}

Lemma 3.6 If the centralizer $Z(m)$ is connected, then the element $g$ in the diagram (3.5) may be taken to be in the normalizer $N_{G} T$ of $T$ in $G$.

Proof Let $g \in G$ be such that

$$
c_{g} m=m^{\prime}
$$

Then $m^{\prime}(A) \subset c_{g}(T) \cap T$, so both $T$ and $c_{g}(T)$ are maximal tori in $Z\left(m^{\prime}\right)$. Since $Z\left(m^{\prime}\right)$ is connected, there is an element $h \in Z\left(m^{\prime}\right)$ such that

$$
c_{h} c_{g}(T)=T,
$$

so $h g \in N_{G} T$. Since $h \in Z\left(m^{\prime}\right)$, we have

$$
m^{\prime}=c_{h} m^{\prime}=c_{h} c_{g} m .
$$

Example 3.3 shows that $Z(m)$ is connected if $G$ is a unitary group. Bott and Samelson have shown ([6]; see Proposition 10.2 of [7]) that $Z(m)$ is connected if $G$ is a spinor group. Indeed they prove the following.

Lemma 3.7 If $G$ is simple and simply connected then the centralizer $Z(m)$ is connected. 


\subsection{Nested fixed-point sets}

Now suppose that $B \supseteq A$ is a larger closed subgroup of $\mathbb{T}$ (primarily, we shall be interested in the case that $B=\mathbb{T}$ ), that $Q / Y$ is a $B$-equivariant principal $G$-bundle, and that $A$ acts trivially on $Y$.

Lemma 3.8 If

$$
m: A \rightarrow T
$$

is a reduction of the action of $A$ on $Q$, then the action of $B$ on $Q$ induces on $Q(m)$ the structure of a $B$-equivariant $Z(m)$-bundle over $Y$. Moreover the Borel construction $Q(m)_{B}$ is a principal $Z(m)$-bundle over $Y_{B}$.

Proof This follows from the fact that $B$ is abelian.

If $F \subseteq Y^{B}$ is a connected component of the subspace of $Y$ fixed by the action of $B$, then we may choose a reduction

$$
m_{F}: B \rightarrow T
$$

of the action of $B$ on $\left.Q\right|_{F}$.

Lemma 3.9 The restriction

$$
\left.m_{F}\right|_{A}: A \rightarrow T
$$

is a reduction of the action of $A$ on $Y$.

Proof The action of $A$ on $Q / Y$ is a section $a$ of $\left(Q \times_{G} \operatorname{hom}\left[A, G^{c}\right]\right) / Y$. The restriction $\left.a\right|_{F}$ records the action of $A$ on $\left.Q\right|_{F}$. The action of $B$ on $\left.Q\right|_{F}$ is a section $b$ of $\left(\left.Q\right|_{F} \times_{G} \operatorname{hom}\left[B, G^{c}\right]\right) / F$, and with the obvious notation we have

$$
\left.b\right|_{A}=\left.a\right|_{F} .
$$

\section{Rational cohomology of principal bundles with compact connected structure group}

Let $E$ be an rational even periodic ring spectrum. Let $G$ be a connected compact Lie group. Let $T$ be a maximal torus of $G$, with Weyl group $W$. The natural isomorphism

$$
\check{T} \otimes \mathbb{T} \rightarrow T
$$


induces a $W$-equivariant isomorphism

$$
\check{T} \otimes P_{E}=\check{T} \otimes \operatorname{sp} E(B \mathbb{T}) \cong \operatorname{sp} E(B T)
$$

of formal groups over $S_{E}$. Moreover [4] the natural map

$$
\operatorname{sp} E(B T) / W \rightarrow \operatorname{sp} E(B G)
$$

is an isomorphism. We shall repeatedly use the resulting isomorphism

$$
\operatorname{sp} E(B G) \cong\left(\check{T} \otimes P_{E}\right) / W .
$$

For example, a principal $G$-bundle $Q$ over $X$ is classified by a map

$$
X \stackrel{Q}{\rightarrow} B G
$$

whose effect in $E$-theory

$$
\operatorname{sp} E(X) \stackrel{\operatorname{sp} E(Q)}{\longrightarrow}\left(\check{T} \otimes P_{E}\right) / W
$$

is a $\operatorname{sp} E(X)$-valued point of $\left(\check{T} \otimes P_{E}\right) / W$.

\subsection{Periodic cohomology of circle-equivariant principal bundles}

We interpret the analysis of section 3 in $E$-theory. Suppose that $G$ is a connected compact Lie group, and that $Q$ is a $\mathbb{T}$-equivariant principal $G$-bundle over a connected $\mathbb{T}$-space $Y$. Suppose that a closed subgroup $A$ of the circle acts trivially on $Y$. Suppose that

$$
m: A \rightarrow T
$$

is a reduction of the action of $A$ on $Q / Y$ with connected centralizer $Z(m)$.

Applying $E$-cohomology to the diagram (3.2) yields

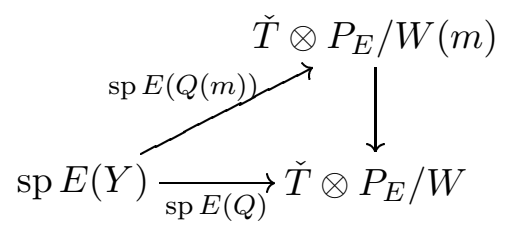

The multiplication

$$
B T \times B T \rightarrow B T
$$

induces the addition map

$$
\left(\check{T} \otimes P_{E}\right) \times\left(\check{T} \otimes P_{E}\right) \stackrel{+}{\longrightarrow} \check{T} \otimes P_{E}
$$


whose restriction to

$$
\left(\check{T} \otimes P_{E}\right)^{W(m)} \times\left(\check{T} \otimes P_{E}\right) \stackrel{+}{\rightarrow} \check{T} \otimes P_{E}
$$

factors to give a translation map

$$
\left(\check{T} \otimes P_{E}\right)^{W(m)} \times\left(\check{T} \otimes P_{E}\right) / W(m) \stackrel{+}{\rightarrow}\left(\check{T} \otimes P_{E}\right) / W(m) .
$$

In $E$ cohomology, Lemma 3.4 implies the following result. It is used in the case $A=\mathbb{T}$ to construct the commutative diagram (8.2) and so prove Lemma 8.4. It is also implies the commutativity of the diagram (10.6), which captures the essence of the "transfer formula" of Bott-Taubes [7]; see Remark 10.7.

Lemma 4.2 The diagram

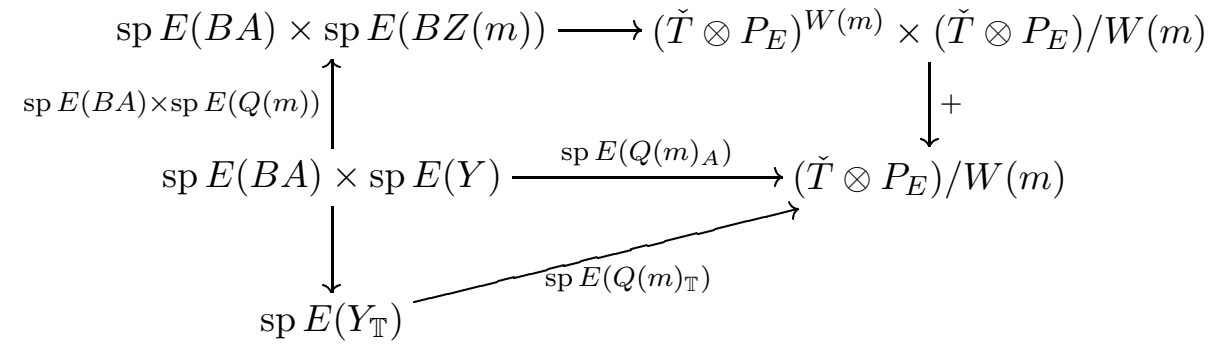

commutes.

\subsection{Holomorphic characteristic classes}

Let $f$ be a $W$-invariant holomorphic function on $\check{T} \otimes \mathbb{G}_{a}^{\text {an }}$. By the splitting principle, the Taylor expansion of $f$ at the origin defines a class in $H P(B G)$. Suppose that $Q$ is a principal $G$-bundle over $X$, with the property that $Q_{\mathbb{T}}$ is a principal $G$-bundle over $X_{\mathbb{T}}$. Then we get a class

$$
f\left(Q_{\mathbb{T}}\right) \in H P\left(X_{\mathbb{T}}\right) .
$$

The following result is due to Rosu.

Lemma 4.3 The class $f\left(Q_{\mathbb{T}}\right)$ is in fact an element of $\mathcal{H}\left(X ; \mathbb{A}_{\mathrm{an}}^{1}\right)$. Similarly, if $f \in\left(\mathcal{O}_{\check{T} \otimes \mathbb{G}_{a}^{\text {an }}}\right)_{0}$, then

$$
f\left(Q_{\mathbb{T}}\right) \in \mathcal{H}(X)_{0}
$$

Proof Proposition A.6 of [19] proves this result in the case that $G=U(n)$ and

$$
f(z)=\prod_{j} g\left(z_{j}\right)
$$


where $g \in \mathcal{O}_{\mathbb{A}_{\text {an }}^{1}, 0}$ and $z=\left(z_{1}, \ldots, z_{n}\right) \in \mathbb{A}_{\text {an }}^{n} \cong \check{T} \otimes \mathbb{G}_{a}^{\text {an }}$. The same argument works in the indicated generality.

An important example of a holomorphic characteristic class is the Euler class associated to a "multiplicative analytic orientation". A power series

$$
f(z)=z+\text { higher terms } \in H P(B \mathbb{T}) \cong \mathbb{C} \llbracket z \rrbracket
$$

satisfying

$$
f(-z)=-f(z)
$$

determines a multiplicative orientation (map of homotopy commutative ring spectra)

$$
\phi: M S O \rightarrow H P,
$$

characterized by the property that if

$$
V=L_{1}+\cdots+L_{d}
$$

is a sum of complex line bundles, then its Euler class is

$$
e_{\phi}(V)=\prod_{j} f\left(c_{1} L_{j}\right) .
$$

If $V$ is an oriented vector bundle, we write $\phi(V) \in H P(V)=H P\left(X^{V}\right)$ for the resulting Thom class. It is multiplicative in the sense that

$$
\phi(V \oplus W)=\phi(V) \wedge \phi(W)
$$

under the isomorphism

$$
(X \times Y)^{V \oplus W} \cong X^{V} \wedge Y^{W}
$$

Definition 4.5 The orientation $\phi$ is analytic if $f$ is contained in the subring $\mathcal{O}_{\mathbb{A}_{\text {an }}^{1}, 0} \subset \mathbb{C} \llbracket z \rrbracket$ of germs of holomorphic functions at 0 ; equivalently, if there is a neighborhood $U$ of 0 in $\mathbb{C}$ on which the power series $f$ converges to a holomorphic function.

Lemma 4.3 implies the following.

Corollary 4.6 [19] If $\phi$ is analytic and $V$ is an oriented $\mathbb{T}$-vector bundle over a compact $\mathbb{T}$-space $X$, then the Euler class $e_{\phi}$ associated to $\phi$ satisfies

$$
e_{\phi}\left(V_{\mathbb{T}}\right) \in \mathcal{H}(X)_{0} .
$$


If $\Phi$ denotes the standard Thom isomorphism, then

$$
\phi\left(V_{\mathbb{T}}\right)=\frac{e_{\phi}\left(V_{\mathbb{T}}\right)}{e_{\Phi}\left(V_{\mathbb{T}}\right)} \Phi\left(V_{\mathbb{T}}\right),
$$

and the ratio of Euler classes is a unit in $\mathcal{H}(X)_{0}$. Of course multiplication by $\Phi\left(V_{\mathbb{T}}\right)$ induces an isomorphism

$$
H\left(X_{\mathbb{T}}\right) \cong H\left(V_{\mathbb{T}}\right),
$$

and so we have the following.

Corollary 4.7 There is a neighborhood $U$ of the origin in $\mathbb{A}_{\text {an }}^{1}$ such that

$$
\phi\left(V_{\mathbb{T}}\right) \in \mathcal{H}(V ; U),
$$

and such that multiplication by this class induces an isomorphism of sheaves

$$
\left.\left.\mathcal{H}(X)\right|_{U} \underset{\phi}{\cong} \mathcal{H}(V)\right|_{U}
$$

In other words, for every open set $U^{\prime} \subseteq U$, multiplication by $\phi\left(V_{\mathbb{T}}\right)$ induces an isomorphism

$$
\mathcal{H}\left(X ; U^{\prime}\right) \stackrel{\phi}{\cong} \mathcal{H}\left(V ; U^{\prime}\right)
$$

Example 4.8 For example, let $\sigma=\sigma(u, q)$ denote the expression

$$
\sigma=\left(u^{\frac{1}{2}}-u^{-\frac{1}{2}}\right) \prod_{n \geq 1} \frac{\left(1-q^{n} u\right)\left(1-q^{n} u^{-1}\right)}{\left(1-q^{n}\right)^{2}} .
$$

This may be considered as an element of $\mathbb{Z} \llbracket q \rrbracket\left[u^{ \pm \frac{1}{2}}\right]$ which is a holomorphic function of $\left(u^{\frac{1}{2}}, q\right) \in \mathbb{C}^{\times} \times D$, where $D=\{q \in \mathbb{C}|0<| q \mid<1\}$. Let $\mathfrak{H}=\{\tau \in$ $\mathbb{C} \mid \Im \tau>0\}$ be the open upper half plane. We may consider $\sigma$ as a holomorphic function of $(z, \tau) \in \mathbb{A}_{\text {an }}^{1} \times \mathfrak{H}$ by

$$
\begin{aligned}
u^{r} & =e^{r z} \\
q^{r} & =e^{2 \pi i r \tau}
\end{aligned}
$$

for $r \in \mathbb{Q}$. It is easy to check using (4.9) that

$$
\begin{aligned}
\sigma(-z) & =-\sigma(z) \\
\sigma(z) & =z+o\left(z^{2}\right) \\
\sigma\left(u q^{n}\right) & =(-1)^{n} u^{-n} q^{-\frac{n^{2}}{2}} \sigma(u) .
\end{aligned}
$$

The equations (4.11) imply that the Taylor expansion of $\sigma$ at the origin defines a multiplicative analytic orientation

$$
M S O \stackrel{\Sigma}{\longrightarrow} H P .
$$


Definition 4.13 If $V$ is an oriented vector bundle, we write $\Sigma(V)$ for the Thom class associated to the orientation (4.12), and $\sigma(V)$ for the associated Euler class.

In $[2$, section 2.7] it is shown that $\Sigma$ is the sigma orientation associated to the elliptic curve $C$ : that is, the diagram

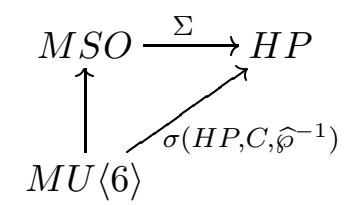

commutes

\section{Degree-four characteristic classes and theta func- tions}

\subsection{Degree-four characteristic classes}

If $G$ is a connected compact Lie group, then by the splitting principle the natural maps

$$
\begin{aligned}
& H^{2}(B G, \mathbb{Z}) \rightarrow H^{2}(B T, \mathbb{Z})^{W} \cong \hat{T}^{W} \\
& H^{4}(B G, \mathbb{Z}) \rightarrow H^{4}(B T, \mathbb{Z})^{W} \cong\left(S^{2} \hat{T}\right)^{W} \cong \operatorname{hom}\left(\Gamma_{2} \check{T}, \mathbb{Z}\right)^{W}
\end{aligned}
$$

are rational isomorphisms. Here, if $M$ is an abelian group, then $S^{2} M$ and $\Gamma_{2} M$ denote degree-two parts of the symmetric and divided power algebras on $M$.

Without rationalizing, a degree-four characteristic class $\xi \in H^{4}(B G, \mathbb{Z})$ gives rise to a homomorphism

$$
\Gamma_{2} \check{T} \stackrel{I}{\rightarrow} \mathbb{Z} .
$$

We shall abuse notation and also write $I$ for the bilinear map

$$
\check{T} \times \check{T} \stackrel{\gamma_{1} \times \gamma_{1}}{\longrightarrow} \Gamma_{2} \check{T} \stackrel{I}{\rightarrow} \mathbb{Z} .
$$

We shall say that the characteristic class $\xi$ is positive definite if the pairing $I$ is so. We also write $\phi$ for the quadratic function given by the composition

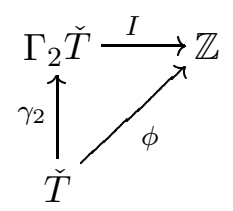


(If $A$ and $B$ are abelian groups, then a function

$$
f: A \rightarrow B
$$

is quadratic if

$$
\begin{aligned}
0 & =f(0) \\
0 & =f(x+y+z)-f(x+y)-f(x+z)-f(y+z)+f(x)+f(y)+f(z) \\
f(-x) & =f(x) .
\end{aligned}
$$

The function

$$
A \stackrel{\gamma_{2}}{\longrightarrow} \Gamma_{2} A
$$

is the universal quadratic function out of $A$ ).

From the definitions it follows that

$$
\begin{aligned}
\phi(a+b) & =\phi(a)+I(a, b)+\phi(b) \\
\phi(n a) & =n^{2} \phi(a) \\
\phi(w a) & =\phi(a) \\
I(w a, w b) & =I(a, b)
\end{aligned}
$$

for $a, b \in \check{T}, n \in \mathbb{Z}$, and $w \in W$.

There are a variety of ways to express the relationship between the characteristic class $\xi$ and the map $I$. For example, suppose that $Q_{0}$ and $Q_{1}$ are two principal $G$-bundles over $X$, given as maps

$$
X \stackrel{Q_{i}}{\longrightarrow} B T .
$$

Then we get a new principal $G$-bundle as the composition

$$
X \stackrel{\Delta}{\longrightarrow} X \times X \stackrel{Q_{0} \times Q_{1}}{\longrightarrow} B T \times B T \stackrel{\mu}{\longrightarrow} B T .
$$

The effect of $Q_{i}$ in cohomology

$$
\operatorname{sp} H \mathbb{Z}(X) \stackrel{\operatorname{sp} H \mathbb{Z}\left(\left(Q_{i}\right)\right)}{\longrightarrow} \operatorname{sp} H \mathbb{Z}(B T) \cong \check{T} \otimes \mathbb{G}_{a} .
$$

is an sp $H \mathbb{Z}(X)$-valued point of $\check{T} \otimes \mathbb{G}_{a}$. Equation (5.3) implies that

$$
\xi\left(\mu\left(Q_{0} \times Q_{1}\right) \Delta\right)=\xi\left(Q_{0}\right)+I\left(\operatorname{sp} H \mathbb{Z}\left(\left(Q_{0}\right)\right), \operatorname{sp} H \mathbb{Z}\left(\left(Q_{1}\right)\right)\right)+\xi\left(Q_{1}\right),
$$

where we have extended $I$ to a bilinear map

$$
\left(\check{T} \otimes \mathbb{G}_{a}\right) \times\left(\check{T} \otimes \mathbb{G}_{a}\right) \rightarrow \mathbb{G}_{a} .
$$

As another example, suppose that $A \subseteq \mathbb{T}$ is a closed subgroup with character group $\mathbb{Z} / n \mathbb{Z}(n \geq 0)$, and suppose that $m: A \rightarrow T$ is a homomorphism. If

$$
\bar{m}, \bar{m}^{\prime} \in \operatorname{hom}(\mathbb{T}, T)=\check{T}
$$


satisfy

$$
\left.\bar{m}\right|_{A}=\left.\bar{m}^{\prime}\right|_{A}=m,
$$

then

$$
\bar{m}^{\prime}=\bar{m}+n \delta
$$

for some $\delta \in \check{T}$, and equation (5.3) implies that

$$
\phi\left(\bar{m}^{\prime}\right) \equiv \phi(\bar{m}) \quad \bmod n
$$

We write $\phi(m)$ for the class of $\phi(\bar{m})$ in $\mathbb{Z} / n$. If $z$ is the chosen generator of $H \mathbb{Z}^{2} B \mathbb{T}$, write also $z$ for the induced generator of $H \mathbb{Z}^{*} B A \cong(\mathbb{Z} / n \mathbb{Z})[z]$. With these conventions

$$
(B m)^{*} \xi=\phi(m) z^{2} .
$$

We write $\hat{I}$ for the map

$$
\hat{I}: \check{T} \rightarrow \hat{T}
$$

which is the adjoint of (5.2). Note that we have

$$
\hat{I}(w a)(w b)=I(w a, w b)=I(a, b)=\hat{I}(a)(b) .
$$

It follows that if $a \in \check{T}^{W}$ then

$$
\hat{I}(a) \in \hat{T}^{W} \rightarrow H^{2}(B G, \mathbb{Q}) .
$$

defines a rational characteristic class of principal $G$-bundles, which we also denote $\hat{I}(a)$.

Now suppose that $Q$ is a $\mathbb{T}$-equivariant principal $G$-bundle over a connected trivial $\mathbb{T}$-space $Y$, so in particular $Q_{\mathbb{T}}$ is a principal $G$-bundle over $Y_{\mathbb{T}}=$ $B \mathbb{T} \times Y$. Let $m \in \check{T}$ be a reduction of the action of $\mathbb{T}$ on $Q / Y$. Then $Q(m)$ is a principal $Z(m)$-bundle over $Y$, and we have the following.

Lemma 5.5 In $H^{4}\left(Y_{\mathbb{T}}, \mathbb{Q}\right)=H^{4}(B \mathbb{T} \times Y, \mathbb{Q})$ we have

$$
\xi\left(Q_{\mathbb{T}}\right)=\phi(m) z^{2}+\hat{I}(m)(Q(m)) z+\xi(Q) .
$$

Proof By the splitting principle, we may suppose that we have a factorization

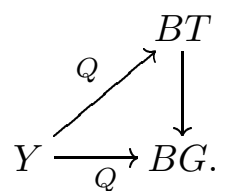

Lemma 3.4 implies that the map

$$
B \mathbb{T} \times Y \stackrel{B m \times Q}{\longrightarrow} B T \times B T \stackrel{\mu}{\longrightarrow} B T
$$


classifies $Q_{\mathbb{T}}$. It follows that

$$
\begin{aligned}
\xi\left(Q_{\mathbb{T}}\right) & =\xi(\operatorname{sp} H \mathbb{Q}(\mu(B m \times Q))) \\
& =\xi(B m)+I(\operatorname{sp} H \mathbb{Q}((B m)), \operatorname{sp} H \mathbb{Q}(Q))+\xi(Q) \\
& =\phi(m) z^{2}+\hat{I}(m)(Q) z+\xi(Q) .
\end{aligned}
$$

Example 5.6 Let $T_{S O(2 d)} \cong \mathbb{T}^{d}$ be the standard maximal torus in $S O(2 d)$ (the image under the map $U(d) \rightarrow S O(2 d)$ of the torus of diagonal matrices), and let $T$ be its preimage in $\operatorname{Spin}(2 d)$. If $m=\left(m_{1}, \ldots, m_{d}\right) \in \mathbb{Z}^{d} \cong\left(T_{S O(2 d)}\right)^{\vee}$, then there is a lift in the diagram

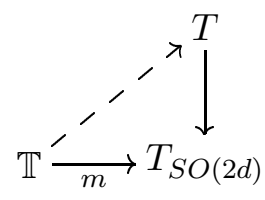

precisely when $\sum m_{i}$ is even, that is

$$
\check{T} \cong\left\{\left(m_{1}, \ldots, m_{d}\right) \in \mathbb{Z}^{d} \mid \sum m_{i} \text { even }\right\} .
$$

The function

$$
\phi\left(m_{1}, \ldots, m_{d}\right)=\frac{1}{2} \sum m_{i}^{2}
$$

therefore defines a quadratic map

$$
\phi: \check{T} \rightarrow \mathbb{Z}
$$

with associated bilinear form

$$
I\left(m, m^{\prime}\right)=\sum m_{i} m_{i}^{\prime} .
$$

It is not hard to check using (5.7) that it is the quadratic form associated to $c_{2} \in H \mathbb{Z}^{4}(B \operatorname{Spin}(2 d))$.

Now suppose that

$$
V=L_{1} \oplus \cdots \oplus L_{d}
$$

is a $\mathbb{T}$-equivariant $\operatorname{Spin}(2 d)$ bundle, written as a sum of $\mathbb{T}$-equivariant complex line bundles, over a trivial $\mathbb{T}$-space $Y$. Let $x_{i}=c_{1} L_{i}$, and suppose that $\mathbb{T}$ acts on $L_{i}$ by the character $m_{i}$. In order that $V_{\mathbb{T}}$ be a spin bundle, we must have

$$
0=w_{2}\left(V_{\mathbb{T}}\right)=\sum_{i}\left(m_{i} z+x_{i}\right) \quad(\bmod 2) .
$$

In that case, Lemma 5.5 says that

$$
c_{2}\left(V_{\mathbb{T}}\right)=\left(\frac{1}{2} \sum m_{i}^{2}\right) z^{2}+\left(\sum m_{i} x_{i}\right) z+c_{2} V
$$

in $H^{4}(Y \times B \mathbb{T})$. 


\subsection{Theta functions}

Recall from section 2.3 that $q=e^{2 \pi i \tau}, \Lambda=2 \pi i \mathbb{Z}+2 \pi i \tau \mathbb{Z}$, and that $C$ is the elliptic curve

$$
C=\mathbb{G}_{a}^{\text {an }} / \Lambda \cong \mathbb{G}_{m}^{\text {an }} / q^{\mathbb{Z}}
$$

Following [16], we define a line bundle over $\check{T} \otimes C$ by the formula

$$
\mathcal{L}=\mathcal{L}(\xi) \stackrel{\text { def }}{=} \frac{\left(\check{T} \otimes \mathbb{G}_{m}^{\text {an }}\right) \times \mathbb{C}}{(u, \lambda) \sim\left(u q^{m}, u^{\hat{I}(m)} q^{\phi(m)} \lambda\right)} .
$$

for $m \in \check{T}$.

Remark 5.9 The identity map of $\left(\check{T} \otimes \mathbb{G}_{m}^{\text {an }}\right) \times \mathbb{C}$ induces for $w \in W$ an isomorphism of line bundles

$$
\mathcal{L} \cong w^{*} \mathcal{L}
$$

over $\check{T} \otimes C$, which is certainly compatible with the multiplication in $W$, so $\mathcal{L}$ descends to a line bundle $\mathcal{A}(\xi)$ on $(\check{T} \otimes C) / W$.

A theta function for $G$ of level $\xi$ is a $W$-invariant holomorphic section of $\mathcal{L}(\xi)$, and so a section of $\mathcal{A}(\xi)$.

Definition 5.10 A theta function for $G$ of level $\xi$ is a function

$$
\theta=\sum_{n>>-\infty} a_{n}(u) q^{n} \in(\mathbb{Z}[\hat{T}])((q))
$$

which for $u=e^{z}$ and $q=e^{2 \pi i \tau}$ is a holomorphic function of $(z, \tau) \in \check{T} \otimes \mathbb{A}_{\text {an }}^{1} \times \mathfrak{H}$, and which satisfies

$$
\begin{aligned}
\theta\left(u q^{m}\right) & =u^{-\hat{I}(m)} q^{-\phi(m)} \theta(u) \\
\theta\left(u^{w}\right) & =\theta(u)
\end{aligned}
$$

for $m \in \check{T}$ and $w \in W$, where $\phi$ and $I$ are the quadratic form and bilinear map associated to the characteristic class $\xi$.

Remark 5.13 Lemma 4.3 implies that a theta function for $G$ gives a holomorphic characteristic class for principal $G$-bundles.

Remark 5.14 There is a good deal of redundancy in the definition. Looijenga studies formal series of the form (5.11) which transform according to (5.12). One has to be careful to identify a group formal series which is closed under the operations implied by (5.12). If $\xi$ is positive definite, then every such formal theta function defines a holomorphic function of $(z, \tau)$ (see [16]). 


\subsection{The sigma function and representations of $L \operatorname{Spin}(2 d)$}

If $G$ is a simple and simply connected Lie group, then there is a unique generator $\xi$ of $H^{4}(B G ; \mathbb{Z}) \cong \mathbb{Z}$ such that the associated pairing $I$ is positive definite. If $\mathcal{V}$ is a representation of $L G$ of level $k$ in the sense of [17], then its character $\chi$ is a theta function for $G$ of level $k \xi$ [14]. The most important example for us is the representation of $L \operatorname{Spin}(2 d)$ whose character is the Euler class of the sigma orientation (4.13); it is a theta function associated to the characteristic class $c_{2}$ of $\operatorname{Spin}(2 d)$.

It is useful to be more explicit about this Euler class. As in Example 5.6, let $T_{S O(2 d)}$ be the image under the map $U(d) \rightarrow S O(2 d)$ of the torus of diagonal matrices. For $u=\left(u_{1}, \ldots, u_{d}\right) \in T$ write

$$
\sigma_{d}(u)=\prod_{i=1}^{d} \sigma\left(u_{i}\right)
$$

The product expression (5.15) and the fact (4.11a) that $\sigma$ is odd imply that $\sigma_{d}\left(u^{w}\right)=\sigma_{d}(u)$ for $w \in W$, and so $\sigma_{d}$ is a $W$-invariant function on $\check{T} \otimes$ $\mathbb{G}_{a}^{\text {an }}$ (with zeroes precisely at the points $\check{T} \otimes \Lambda$ ). By Lemma 4.3 it defines a holomorphic characteristic class for oriented vector bundles of rank $2 d$. If $V$ is such a vector bundle, then $\sigma(V)=\sigma_{d}(V)$.

The fractional powers of $u$ in the expression (4.9) for $\sigma$ prevent $\sigma_{d}$ from being a theta function for $S O(2 d)$, but if $G=\operatorname{Spin}(2 d)$ then the formula

$$
\sigma_{d}(u)=(-1)^{d}\left(\prod_{i} u_{i}\right)^{\frac{1}{2}} \prod_{i}\left(\left(1-u_{i}\right) \prod_{n \geq 1} \frac{\left(1-q^{n} u_{i}\right)\left(1-q^{n} u_{i}^{-1}\right)}{\left(1-q^{n}\right)^{2}}\right)
$$

shows that $\sigma_{d} \in(\mathbb{Z}[\hat{T}]) \llbracket q \rrbracket$. The formula (4.11c) implies that, if $I$ and $\phi$ are the pairing and quadratic form associated to the generator $c_{2} \in H^{4}(B G ; \mathbb{Z})$ as in Example 5.6, then

$$
\sigma_{d}\left(u q^{m}\right)=u^{-\hat{I}(m)} q^{-\phi(m)} \sigma_{d}(u),
$$

so $\sigma_{d}$ is a theta function for $\operatorname{Spin}(2 d)$ of level $c_{2}$. Up to the factor $\prod_{n}\left(1-q^{n}\right)^{2 d}$, it is the character of an irreducible representation of $L \operatorname{Spin}(2 d)$ of level 1 $[14,17,15]$.

\subsection{A useful holomorphic characteristic class}

Suppose that $Q$ is a $\mathbb{T}$-equivariant principal $G$-bundle over a connected $\mathbb{T}$-space $Y$. Suppose that $\mathbb{T}[n] \subset \mathbb{T}$ acts trivially on $Y$. Let

$$
m: \mathbb{T}[n] \rightarrow T
$$


be a reduction of the action of $\mathbb{T}[n]$ on $Q / Y$.

Let $\xi \in H^{4}(B G ; \mathbb{Z})$ be a positive definite class, with associated quadratic form $\phi$ and bilinear map $I$. Let $\theta$ be a theta function of level $\xi$ for $G$ (Definition 5.10). Recall that

$$
C=\mathbb{G}_{a}^{\text {an }} /(2 \pi i \mathbb{Z}+2 \pi i \tau \mathbb{Z}),
$$

and let $a$ be a point of $C$ of order $n$. Choose a point $\bar{a} \in \mathbb{G}_{a}^{\text {an }}$ such that $\wp(\bar{a})=a$.

We are going to define a holomorphic function

$$
F=F(\theta, m, \bar{a}) \in \mathcal{O}\left(\check{T} \otimes \mathbb{G}_{a}^{\mathrm{an}}\right)^{W(m)},
$$

and so a holomorphic characteristic class of principal $Z(m)$-bundles (see section 4.2). To give a formula for $F$ it is convenient to define

$$
\alpha=e^{2 \pi i \bar{a}},
$$

and recall (2.1) that we have set

$$
\begin{aligned}
q^{r} & =e^{2 \pi i r \tau} \\
u^{r} & =e^{r z}
\end{aligned}
$$

for $r \in \mathbb{Q}$.

Since $n a=0$ in $C$, there are unique integers $\ell$ and $k$ such that

$$
n \bar{a}=2 \pi i \ell+2 \pi i \tau k .
$$

We choose an extension $\bar{m}$ making the diagram<smiles>[Al]1[In][Te]1</smiles>

commute. With these choices, the formula for $F$ is

$$
F(z)=u^{\frac{k}{n} \hat{I}(\bar{m})} \alpha^{\frac{k}{n} \phi(\bar{m})} \theta\left(u \alpha^{\bar{m}}\right) .
$$

Remark 5.17 The factors preceding the $\theta$ are closely related to the line bundle $\mathcal{V}^{\frac{1}{n}}$ which appears in [7] and [1].

Lemma 5.18 $F$ is independent of the choice of lift $\bar{m}$. 
Proof Let $\bar{m}^{\prime}$ be another choice. Let $F^{\prime}$ be the function defined using $\bar{m}^{\prime}$. Since $\bar{m}^{\prime}$ and $\bar{m}$ both restrict to $m$ on $A$, there is a $\Delta \in \check{T}$ such that

$$
\bar{m}^{\prime}=\bar{m}+n \Delta \text {. }
$$

We have

$$
\begin{aligned}
F^{\prime}(z) & =u^{\frac{k}{n} \hat{I}\left(\bar{m}^{\prime}\right)} \alpha^{\frac{k}{n} \phi\left(\bar{m}^{\prime}\right)} \theta\left(u \alpha^{\bar{m}^{\prime}}\right) \\
& =u^{\frac{k}{n} \hat{I}(\bar{m}+n \Delta)} \alpha^{\frac{k}{n} \phi(\bar{m}+n \Delta)} \theta\left(u \alpha^{\bar{m}} q^{k \Delta}\right) \\
& =u^{\frac{k}{n} \hat{I}(\bar{m})} u^{k \hat{I}(\Delta)} \alpha^{\frac{k}{n} \phi(\bar{m})} \alpha^{k I(\bar{m}, \Delta)} q^{k^{2} \phi(\Delta)} u^{-k \hat{I}(\Delta)} \alpha^{-k I(\Delta, \bar{m})} q^{-k^{2} \phi(\Delta)} \theta\left(u \alpha^{\bar{m}}\right) \\
& =F(z) .
\end{aligned}
$$

Lemma 5.19 $F$ is invariant under $W(m)$.

Proof Suppose $w \in W(m)$. We have

$$
w m=m
$$

so

$$
\bar{m}=w \bar{m}+n \Delta
$$

for some $\Delta \in \check{T}$. The proof is now similar to the proof of Lemma 5.18.

The dependence of $F(\theta, m, \bar{a})$ on the lift $\bar{a}$ can be calculated as follows. Let $\bar{a}^{\prime}$ be another lift. Then

$$
\bar{a}^{\prime}=\bar{a}+\lambda
$$

for some $\lambda \in 2 \pi i \mathbb{Z}+2 \pi i \tau \mathbb{Z}$, so letting

$$
\beta=e^{\bar{a}^{\prime}}
$$

we have

$$
\beta=\alpha q^{\delta}
$$

for some $\delta \in \mathbb{Z}$. Thus

$$
\beta^{n}=q^{k^{\prime}}
$$

with $k^{\prime}=k+n \delta$, and so the quantity

$$
w\left(a, q^{\frac{1}{n}}\right) \stackrel{\text { def }}{=} \alpha^{-1} q^{\frac{k}{n}}=\beta^{-1} q^{\frac{k^{\prime}}{n}}
$$

is an $n^{\text {th }}$ root of unity which is independent of the choice of lift of $a$; in fact it is the Weil pairing of $a$ and $q^{\frac{1}{n}}$ in the curve $C$. Because it is an $n^{\text {th }}$ root of unity, the quantity

$$
w\left(a, q^{\frac{1}{n}}\right)^{\phi(m)} \stackrel{\text { def }}{=} w\left(a, q^{\frac{1}{n}}\right)^{\phi(\bar{m})}
$$

is independent of the lift $\bar{m}$ of $m$. Using the functional equation for $\theta$ (5.12) it is straightforward to check the following. 


\section{Lemma 5.20}

$$
F\left(\theta, m, \bar{a}^{\prime}\right)=w\left(a, q^{\frac{1}{n}}\right)^{\delta \phi(m)} F(\theta, m, \bar{a}) .
$$

Lemma 5.19 implies that the Taylor series expansion of $F(\theta, m, \bar{a})$ defines a class in $\hat{H}(B Z(m))=\hat{H}(B T)^{W(m)}$, which we also denote $F(\theta, m, \bar{a})$. Let $\theta(Q, m, \bar{a}) \in \mathcal{H}\left(Y ; \mathbb{A}_{\text {an }}^{1}\right)$ be the holomorphic cohomology class given by the formula

$$
\theta(Q, m, \bar{a})=Q(m)_{\mathbb{T}}^{*} F(\theta, m, \bar{a})
$$

(using Lemma 4.3 to conclude that the class $\theta(Q, m, \bar{a})$ is in fact holomorphic).

Lemma 5.22 If the centralizer $Z(m)$ is connected, for example if $G$ is unitary or spin, then the class $\theta(Q, m, \bar{a})$ is independent of the reduction $m$ of the action of $\mathbb{T}[n]$ on $Q / Y$.

Proof Let $m^{\prime}$ be another reduction of the action of $\mathbb{T}[n]$ on $Q / Y$. By Lemma 3.6, there is an element $w \in W$ such that

$$
m^{\prime}=w m \text {. }
$$

If $\bar{m}: \mathbb{T} \rightarrow T$ is a lift of $m$, then $w \bar{m}$ is a lift of $m^{\prime}$. Using this lift to define $F\left(\theta, m^{\prime}, \bar{a}\right)$, we have

$$
\begin{aligned}
w^{*} F\left(\theta, m^{\prime}, \bar{a}\right)(z) & =F\left(\theta, m^{\prime}, \bar{a}\right)(w(z)) \\
& =\left(u^{w}\right)^{\frac{k}{n} \hat{I}(w \bar{m})} \alpha^{\frac{k}{n} \phi(w \bar{m})} \theta\left(u^{w} \alpha^{w \bar{m}}\right) \\
& =u^{\frac{k}{n} \hat{I}(\bar{m})} \alpha^{\frac{k}{n} \phi(\bar{m})} \theta\left(u \alpha^{\bar{m}}\right) \\
& =F(\theta, m, \bar{a})(z) .
\end{aligned}
$$

Since the diagram

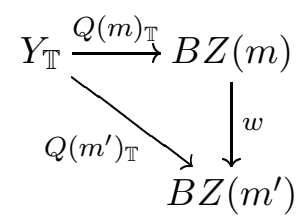

commutes, we have

$$
\begin{aligned}
\theta\left(Q, m^{\prime}, \bar{a}\right) & =Q\left(m^{\prime}\right)_{\mathbb{T}}^{*} F\left(\theta, m^{\prime}, \bar{a}\right) \\
& =Q(m)_{\mathbb{T}}^{*} w^{*} F\left(\theta, m^{\prime}, \bar{a}\right) \\
& =Q(m)_{\mathbb{T}}^{*} F(\theta, m, \bar{a}) \\
& =\theta(Q, m, \bar{a}) .
\end{aligned}
$$

The results of this section justify the following. 
Definition 5.23 Suppose that $Q$ is a $\mathbb{T}$-equivariant principal $G$-bundle over a connected $\mathbb{T}$-space $Y$ on which $\mathbb{T}[n]$ acts trivially. Suppose that for some (equivalently every) reduction

$$
m: \mathbb{T}[n] \rightarrow T
$$

of the action of $\mathbb{T}[n]$ on $Q / Y$, the centralizer $Z(m)$ is connected. Let $C$ be the elliptic curve $\mathbb{G}_{a}^{\text {an }} / 2 \pi i \mathbb{Z}+2 \pi i \tau \mathbb{Z}$. Let $a$ be a point of $C$ of order $n$. Let $\theta$ be a theta function for $G$ of level $\xi$, and let $\bar{a}$ be a point of $\mathbb{A}_{\text {an }}^{1}$ whose image in $C$ is $a$. We define $\theta(Q, \bar{a}) \in \mathcal{H}\left(Y ; \mathbb{A}_{\text {an }}^{1}\right)$ to be the holomorphic cohomology class

$$
\theta(Q, \bar{a})=\theta(Q, m, \bar{a})
$$

in particular this class does not depend on the choice of $m$.

Lemma 5.25 If $\bar{a}^{\prime}=\bar{a}+2 \pi i s+2 \pi i \delta \tau$ is another lift of $a$, then

$$
\theta\left(Q, \bar{a}^{\prime}\right)=w\left(a, q^{\frac{1}{n}}\right)^{\delta \phi(m)} \theta(Q, \bar{a}) ;
$$

again this class does not depend on the choice of $m$.

An important case of the preceding constructions is that $G=\operatorname{Spin}(2 d)$, and $\sigma_{d}$ is the character of the basic representation of $L G$ as in section 5.3. Let $p: G \rightarrow S O(2 d)$ be standard the double cover. Let $P / Y$ be the resulting $\mathbb{T}$ equivariant $S O(2 d)$-bundle over $Y$, and let $V$ be the associated vector bundle. We recall from [7] that Lemma 3.7 implies that the sub-vector bundle $V^{\mathbb{T}[n]}$ is orientable. Explicitly, if $m$ is a reduction of the action of $\mathbb{T}[n]$ on $Q / Y$, then $p m$ is a reduction of the action of $\mathbb{T}[n]$ on $P / Y$. If $V^{\mathbb{T}[n]}$ has rank $2 k$, then the map

$$
Y \rightarrow B O(2 k)
$$

classifying $V^{\mathbb{T}[n]}$ factors through the map $Y \rightarrow B Z(p m)$ classifying $P(p m)$, and we have the solid diagram

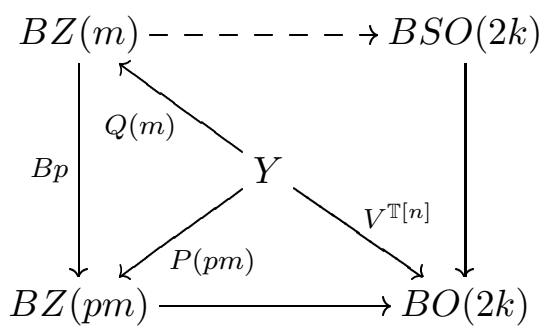

Since (by Lemma 3.7) the centralizer $Z(m)$ is connected, there is a dotted arrow making the diagram commute. In other words, $V^{\mathbb{T}[n]}$ is orientable, and a choice of orientation $\epsilon\left(V^{\mathbb{T}[n]}\right)$ determines a map

$$
f: B Z(m) \rightarrow B S O(k)
$$


such that $f Q(m)$ classifies $\left(V^{\mathbb{T}[n]}, \epsilon\left(V^{\mathbb{T}[n]}\right)\right)$. In other words, Lemma 3.7 implies the following result (as I learned from [7]).

Lemma 5.27 If $V$ is a $\mathbb{T}$-equivariant $\operatorname{Spin}(2 d)$-vector bundle, then it is $\mathbb{T}$ orientable.

Let $\sigma_{k} \in \hat{H}(B S O(2 k))$ be the characteristic class associated to the sigma function as in section 5.3. We then have two $W(m)$-invariant holomorphic functions on $\check{T} \otimes \mathbb{G}_{a}^{\text {an }}$. One is $f^{*} \sigma_{k}$, and the other is $F(\theta, m, \bar{a})$.

Lemma 5.28 The ratio

$$
R=\frac{f^{*} \sigma_{k}}{F\left(\sigma_{d}, m, \bar{a}\right)}
$$

is a $W(m)$-invariant unit of $\left(\mathcal{O}_{\check{T} \otimes \mathbb{G}_{a}^{a n}}\right)_{0}$.

Proof The poles of $R$ occur at zeroes of $F$. Using the standard maximal torus of $S O(2 d)$ we may write a typical element of $\check{T} \otimes \mathbb{G}_{m}^{\text {an }}$ as

$$
\left(u_{1}, \ldots, u_{d}\right) \in\left(\mathbb{G}_{m}^{\text {an }}\right)^{d} \cong \check{T} \otimes \mathbb{G}_{m}^{\text {an }} .
$$

In these terms, a lift $\bar{m}$ of $m$ is of the form

$$
u^{\bar{m}}=\left(u^{m_{1}}, \ldots, u^{m_{d}}\right)
$$

for some integers $m_{1}, \ldots, m_{d}$. We have

$$
F\left(\sigma_{d}, m, \bar{a}\right)=u^{\frac{k}{n} \hat{I}(\bar{m})} \alpha^{\frac{k}{n} \phi(\bar{m})} \prod_{j=1}^{d} \sigma\left(u_{j} \alpha^{m_{j}}\right) .
$$

The product of sigma functions contributes a zero near $z=0$ if and only if $m_{j} a=0$ in $C$. Let $j_{1}, \ldots, j_{k} \in\{1, \ldots, d\}$ be the indices such that $m_{j_{i}} a=0$ in $C$; then

$$
f^{*} \sigma_{k}=\prod_{i=1}^{k} \sigma\left(u_{j_{i}} \alpha^{m_{j_{i}}}\right) .
$$

So the zeroes of $f^{*} \sigma_{k}$ precisely cancel those of $F\left(\sigma_{d}, m, \bar{a}\right)$.

Lemmas 4.3 and 5.28 together imply that $R$ defines a holomorphic characteristic class for $Z(m)$-bundles.

Corollary 5.29 The holomorphic characteristic class

$$
R\left(V, V^{\mathbb{T}[n]}, \epsilon\left(V^{\mathbb{T}[n]}\right), \bar{a}\right) \stackrel{\text { def }}{=} Q(m)_{\mathbb{T}}^{*} R \in\left(\mathcal{H}(Y)_{0}\right)^{\times}
$$

is independent of the reduction $m$, and satisfies

$$
e_{\sigma}\left(V_{\mathbb{T}}^{\mathbb{T}[n]}, \epsilon\left(V^{\mathbb{T}[n]}\right)\right)=R\left(V, V^{\mathbb{T}[n]}, \epsilon\left(V^{\mathbb{T}[n]}\right), \bar{a}\right) \sigma(V, \bar{a}) .
$$




\section{Equivariant elliptic cohomology}

\subsection{Adapted open cover of an elliptic curve}

If $X$ is a $\mathbb{T}$-space and if $a$ is a point of $C$, then we define

$$
X^{a}= \begin{cases}X^{\mathbb{T}[k]} & a \text { is of order exactly } k \text { in } C \\ X^{\mathbb{T}} & \text { otherwise. }\end{cases}
$$

Let $N \geq 1$ be an integer.

Definition 6.1 A point $a \in C$ is special for $X$ if $X^{a} \neq X^{\mathbb{T}}$.

If $V$ is a $\mathbb{T}$-bundle over a $\mathbb{T}$-space $X$, then it is convenient to consider a few additional points to be special. Suppose that $F$ is a component of $X^{\mathbb{T}}$ and

$$
m: \mathbb{T} \rightarrow T
$$

is a reduction of the action of $\mathbb{T}$ on the principal bundle associated to $V$. If we choose an isomorphism

$$
\check{T} \cong \mathbb{Z}^{r}
$$

then we may view $m$ as an array of integers $\left(m_{1}, m_{2}, \ldots, m_{r}\right)$. These integers are called the exponents or rotation numbers of $V$ at $F$. Let $V^{+}$denote the one-point compactification of $V$.

Definition 6.2 A point $a$ in $C$ is special for $V$ if it is special for $V^{+}$or if for some component $F$ of $X^{\mathbb{T}}$ there is a rotation number $m_{j}$ of $V$ such that $m_{j} a=0$.

In either case, if $X$ is a finite $\mathbb{T}$-CW complex, then the set of special points is a finite subset of the torsion subgroup of $C$.

Definition 6.3 An indexed open cover $\left\{U_{a}\right\}_{a \in C}$ of $C$ is adapted to $X$ or $V$ if it satisfies the following.

1) $a$ is contained in $U_{a}$ for all $a \in C$.

2) If $a$ is special and $a \neq b$, then $a \notin U_{a} \cap U_{b}$.

3) If $a$ and $b$ are both special and $a \neq b$, then the intersection $U_{a} \cap U_{b}$ is empty.

4) If $b$ is ordinary, then $U_{a} \cap U_{b}$ is non-empty for at most one special $a$.

5) Each $U_{a}$ is small (2.2).

Lemma 6.4 Let $X$ be a finite $\mathbb{T}-C W$ complex. Then $C$ has an adapted open cover, and any two adapted open covers have a common refinement. 


\subsection{Complex elliptic cohomology}

Let

$$
\hat{E} \stackrel{\text { def }}{=}\left(H P, C, \widehat{\wp}^{-1}\right)=\left(H P, C, \log _{\omega}\right)
$$

be the elliptic spectrum associated to the elliptic curve $C$ (see (2.10)). Note that this is just a form of ordinary cohomology. The hat in the notation indicates that this is a completion of the associated holomorphic cohomology. Namely, suppose that $U \subset C$ is a small open neighborhood of the identity in $C$. Suppose that $V \subset \mathbb{A}_{\text {an }}^{1}$ is the component of $\wp^{-1} U$ containing the origin. Let $\left.\operatorname{sp} E\left(X_{\mathbb{T}}\right)\right|_{U}=\left(U,\left.E\left(X_{\mathbb{T}}\right)\right|_{U}\right)$ be the ringed space defined as the pull-back in the diagram

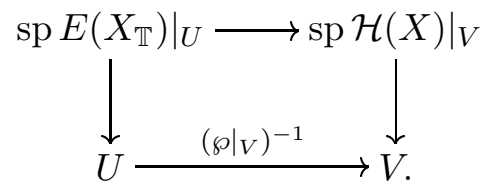

The diagram (2.9) shows that $\operatorname{sp} \hat{E}\left(X_{\mathbb{T}}\right)$ and $\left.\operatorname{sp} E\left(X_{\mathbb{T}}\right)\right|_{U}$ are related by the formula

$$
\operatorname{sp} \hat{E}\left(X_{\mathbb{T}}\right) \cong\left(\left.\operatorname{sp} E\left(X_{\mathbb{T}}\right)\right|_{U}\right)_{0}^{\wedge} \text {. }
$$

\subsection{Equivariant elliptic cohomology}

Grojnowski's circle-equivariant extension of $\hat{E}$ is a contravariant functor associating to a compact $\mathbb{T}$-manifold $X$ a $\mathbb{Z} / 2$-graded $\mathcal{O}_{C}$-algebra $E_{\mathbb{T}}(X)$, with the property that

$$
E_{\mathbb{T}}(*)=\mathcal{O}_{C} .
$$

It is equipped with a natural isomorphism

$$
\hat{E}\left(X_{\mathbb{T}}\right) \stackrel{A(X)}{\cong}\left(E_{\mathbb{T}}(X)\right)_{0}^{\wedge},
$$

such that

$$
A(*)=\log _{\omega}:\left(\mathcal{O}_{C}\right)_{0}^{\wedge} \cong \hat{E}(B \mathbb{T}) .
$$

We shall write $\operatorname{sp} E_{\mathbb{T}}(X)$ for the ringed space $\left(C, E_{\mathbb{T}}(X)\right)$ (see $(2.5)$ ). We take this opportunity to phrase the account in [1] of the construction of $E_{\mathbb{T}}(X)$ as the construction of a covariant functor

$$
X \mapsto \operatorname{sp} E_{\mathbb{T}}(X)
$$

from finite $\mathbb{T}-\mathrm{CW}$ complexes to ringed spaces (2.5) over $C$, equipped with an identification

$$
\operatorname{sp} E_{\mathbb{T}}(*)=C
$$


and a natural isomorphism of formal schemes

$$
\operatorname{sp} \hat{E}\left(X_{\mathbb{T}}\right) \stackrel{A(X)}{\cong}\left(\operatorname{sp} E_{\mathbb{T}}(X)\right)_{0}^{\wedge}
$$

such that

$$
A(*)=\log _{\omega}: P_{\hat{E}}=\widehat{\mathbb{G}}_{a} \cong \widehat{C} .
$$

If $\mathcal{X}=\left(X, \mathcal{O}_{X}\right)$ is a ringed space and $U$ is an open set of $X$, then we may write $\mathcal{X}(U)$ in place of $\mathcal{O}_{X}(U)$.

Let $\left\{U_{a}\right\}_{a \in C}$ be an adapted open cover of $C$. For each $a \in C$, we make a ringed space sp $E_{\mathbb{T}, a}(X)=\left(U_{a}, E_{\mathbb{T}, a}(X)\right)$ over $U_{a}$ as the pull back in the diagram

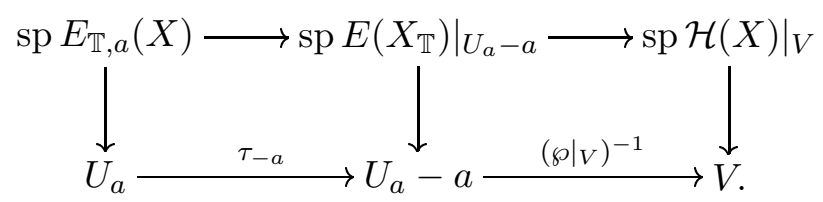

As in (6.5), $V$ is the component of $\wp^{-1}\left(U_{a}-a\right)$ containing the origin. In other words, let $V_{a} \subset \wp^{-1}\left(U_{a}\right)$ be the component containing the origin. For $U \subset U_{a}$ let $V=V_{a} \cap \wp^{-1}(U-a)$, and let

$$
E_{\mathbb{T}, a}(X)(U)=\mathcal{H}\left(X^{a} ; V\right),
$$

considered as an $\mathcal{O}_{C}(U)$-algebra via the isomorphism

$$
U \stackrel{\tau_{-a}}{\longrightarrow} U-a \stackrel{\left(\left.\wp\right|_{V}\right)^{-1}}{\longrightarrow} V .
$$

If $a \neq b$ and $U_{a} \cap U_{b}$ is not empty, then by the definition (6.3) of an adapted cover, at least one of $U_{a}$ and $U_{b}$, suppose $U_{b}$, contains no special point. In particular we have $X^{b}=X^{\mathbb{T}}$ and so an isomorphism

$$
\left.\operatorname{sp} E\left(X_{\mathbb{T}}^{b}\right)\right|_{U} \cong \operatorname{sp} E\left(X^{b}\right) \times U
$$

i.e.

$$
E\left(X_{\mathbb{T}}^{b}\right) \underset{\mathbb{C}[z]}{\otimes} \mathcal{O}_{U} \cong E\left(X^{b}\right) \underset{\mathbb{C}}{\otimes} \mathcal{O}_{U}
$$

for any small neighborhood $U$ of the origin.

Lemma 6.8 If $a \neq b, U \subset U_{a} \cap U_{b}$, and $b$ is not special, then the inclusion

$$
i: X^{b} \rightarrow X^{a}
$$

induces an isomorphism

$$
\left.\left.\operatorname{sp} E\left(X_{\mathbb{T}}^{b}\right)\right|_{U-a} \cong \operatorname{sp} E\left(X_{\mathbb{T}}^{a}\right)\right|_{U-a} .
$$


Proof If $a$ is not special, then $X^{a}=X^{b}$ and the result is obvious. If $a$ is special, then it is not contained in $U$ (by the definition of an adapted cover), and so 0 is not contained in $U-a$. The localization theorem (2.7) gives the result.

Let $U=U_{a} \cap U_{b}$. We define

$$
\psi_{a b}=\psi_{a b}^{X}:\left.\left.\operatorname{sp} E_{\mathbb{T}, a}(X)\right|_{U} \cong \operatorname{sp}_{\mathbb{T}, b}(X)\right|_{U}
$$

as the arrow making the diagram

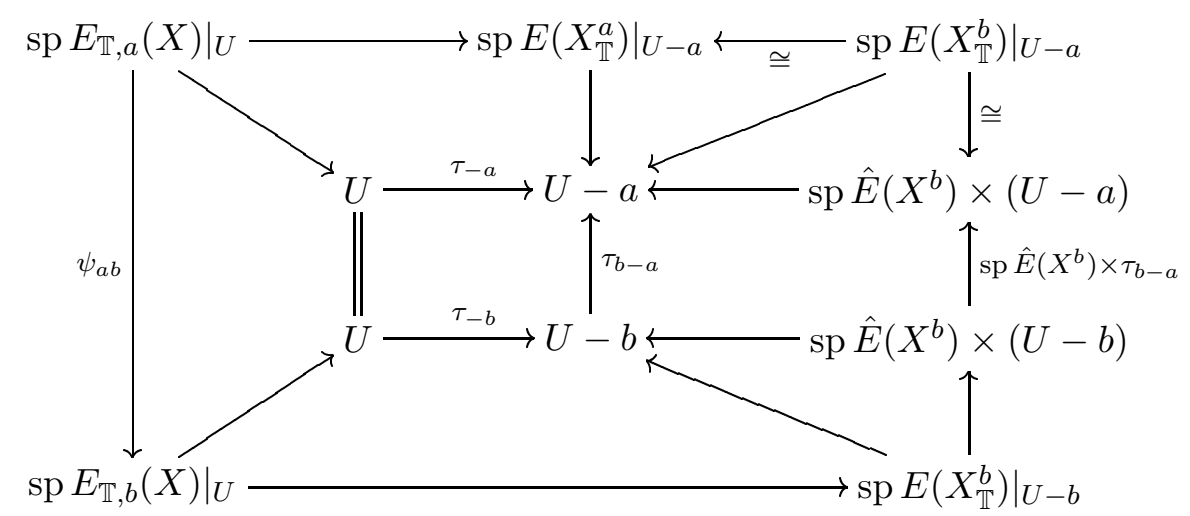

commute. The cocycle condition

$$
\psi_{b c} \psi_{a b}=\psi_{a c}
$$

needs to be checked only when two of $a, b, c$ are not special; and in that case it follows easily from the equation

$$
\tau_{c-b} \tau_{b-a}=\tau_{c-a} .
$$

We shall write $\operatorname{sp} E_{\mathbb{T}}(X)$ for the ringed space over $C$, and $E_{\mathbb{T}}(X)$ for its structure sheaf. One then has the following ([11]; for a published account see [19]).

Proposition 6.10 $\operatorname{sp} E_{\mathbb{T}}(X)$ is a ringed space over $C$, which is independent up to canonical isomorphism of the choice of adapted open cover.

\section{Equivariant elliptic cohomology of Thom spaces}

Suppose that $V$ is a $\mathbb{T}$-equivariant vector bundle over $X$, and let $V_{0}$ be the complement of the zero section of $V$. We abbreviate as

$$
E_{\mathbb{T}}(V)=E_{\mathbb{T}}\left(V, V_{0}\right)
$$


the $E_{\mathbb{T}}(X)$-module associated to the reduced $E_{\mathbb{T}}$-cohomology of the Thom space of $V$. Explicitly, for each point $a \in C$ we define a sheaf $E_{\mathbb{T}, a}(V)$ of $E_{\mathbb{T}, a}(X)$-modules as the pull-back

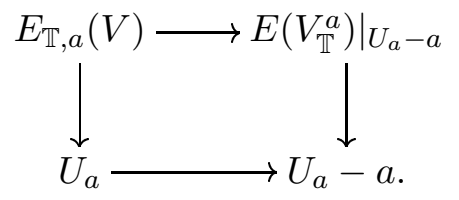

For $a$ special and $b$ not with $U_{a} \cap U_{b}$ non-empty, the isomorphism

$$
\psi_{a b}^{V}:\left.\left.E_{\mathbb{T}, a}(V)\right|_{U_{a} \cap U_{b}} \cong E_{\mathbb{T}, b}(V)\right|_{U_{a} \cap U_{b}}
$$

is given by

$$
\begin{aligned}
\left.\left.E\left(V_{\mathbb{T}}^{a}\right)\right|_{U_{a b}-a}(U-a) \underset{\cong}{\cong}\left(V_{\mathbb{T}}^{b}\right)\right|_{U_{a b}-a}(U-a) \cong E\left(V^{b}\right) \underset{\mathbb{C}}{\otimes} \mathcal{O}(U-a) \stackrel{\tau_{b-a}^{*}}{\longrightarrow} \\
\left.E\left(V^{b}\right) \underset{\mathbb{C}}{\otimes} \mathcal{O}(U-b) \cong E\left(V_{\mathbb{T}}^{b}\right)\right|_{U_{a b}-b}(U-b) ;
\end{aligned}
$$

where $U_{a b}=U_{a} \cap U_{b} ; U$ denotes an open subset of $U_{a b}$, and we have omitted a pullback (7.2) at either end.

Definition 7.3 The vector bundle $V$ is $\mathbb{T}$-orientable if for each closed subgroup $A \subseteq \mathbb{T}$, the fixed bundle $V^{A}$ over $X^{A}$ is orientable. A $\mathbb{T}$-orientation $\epsilon$ on $V$ is a choice $\epsilon\left(V^{A}\right)$ of orientation on $V^{A}$ for each $A$.

Lemma 7.4 If $V$ is a $\mathbb{T}$-orientable vector bundle over $X$, then $E_{\mathbb{T}}(V)$ is a line bundle over $\operatorname{sp} E_{\mathbb{T}}(X)$, that is, $E_{\mathbb{T}}(V)$ is an invertible $E_{\mathbb{T}}(X)$-module.

Proof If $V$ is $\mathbb{T}$-orientable, then the Thom isomorphism in ordinary cohomology implies that each $E_{\mathbb{T}, a}(V)$ is an invertible $E_{\mathbb{T}, a}(X)$-module.

We recall from $[19,1]$ the construction of an explicit cocycle for this line bundle. Let $\phi$ be a multiplicative analytic orientation (4.5), and let $\epsilon$ be a $\mathbb{T}$-orientation on $V$.

Definition 7.5 An indexed open cover $\left\{U_{a}\right\}_{a \in C}$ of $C$ is adapted to $(V, \phi, \epsilon)$ if it is adapted to $V$ (see Definition 6.3), and if for every point $a \in C$, the equivariant Thom class $\phi\left(V_{\mathbb{T}}^{a}, \epsilon\right)$ induces an isomorphism

$$
\mathcal{H}\left(X ; U_{a}-a\right) \stackrel{\phi}{\cong} \mathcal{H}\left(V^{a} ; U_{a}-a\right) .
$$


Corollary 4.7 implies that $C$ has an indexed open cover adapted to $(V, \phi, \epsilon)$. Choose such a cover. Suppose that $a, b$ are two points of $C$, such that $U=$ $U_{a} \cap U_{b}$ is non-empty: we may suppose that $a=b$ or that $b$ is not special. Consider the case that $b$ is not special. Let $\bar{U}_{a} \subset \mathbb{A}_{\text {an }}^{1}$ be the component of the preimage of $U_{a}-a$ containing the origin, and let

$$
\left.\wp\right|_{\bar{U}_{a}}: \bar{U}_{a} \rightarrow U_{a}-a
$$

be the induced isomorphism. Let $\bar{U} \subset \bar{U}_{a}$ be the preimage of $U$. Let

$$
j:\left(V^{b}, V_{0}^{b}\right) \rightarrow\left(V^{a}, V_{0}^{a}\right)
$$

be the natural map. The Localization Theorem (Theorem 2.7) implies that the ratio of Euler classes

$$
e_{\phi}\left(V^{a}, V^{b}, \epsilon\right) \stackrel{\text { def }}{=} \frac{j^{*} \phi\left(\left(V^{a}\right)_{\mathbb{T}}, \epsilon\left(V^{a}\right)\right)}{\phi\left(\left(V^{b}\right)_{\mathbb{T}}, \epsilon\left(V^{b}\right)\right)}
$$

is a unit of $\mathcal{H}\left(X^{\mathbb{T}} ; \bar{U}\right)$.

Recall that there are tautological isomorphisms

$$
\left.E_{\mathbb{T}, a}(X)(U) \stackrel{\tau_{a}^{*}}{\cong} E\left(X_{\mathbb{T}}^{a}\right)\right|_{U-a}(U-a) \stackrel{\left(\left.\wp\right|_{\bar{U}}\right)^{*}}{\cong} \mathcal{H}\left(X^{a} ; \bar{U}\right) .
$$

Let

$$
e_{\phi}(a, b, \epsilon) \in E_{\mathbb{T}, a}(X)(U)^{\times}
$$

be given by the formula

$$
\left(\left.\wp\right|_{\bar{U}}\right)^{*} \tau_{a}^{*} e_{\phi}(a, b, \epsilon)=e_{\phi}\left(V^{a}, V^{b}, \epsilon\right) .
$$

Note that $e_{\phi}(a, b, \epsilon)=1$ if neither $a$ nor $b$ is special; we also set

$$
e_{\phi}(a, a, \epsilon)=1
$$

for all $a$. It is easy to check that

$$
\psi_{b c}^{X}\left(\psi_{a b}^{X}\left(e_{\phi}(a, b, \epsilon)\right) e_{\phi}(b, c, \epsilon)\right)=\psi_{a c}^{X} e_{\phi}(a, c, \epsilon)
$$

if $U_{a} \cap U_{b} \cap U_{c}$ is non-empty (since in that case at least two of $a, b, c$ are ordinary). Thus the $e_{\phi}(a, b)$ define a cohomology class $[\phi, V, \epsilon] \in H^{1}\left(C ; E_{\mathbb{T}}(X)^{\times}\right)$. Let $E_{\mathbb{T}}(X)^{[\phi, V, \epsilon]}$ be the resulting invertible sheaf of $E_{\mathbb{T}}(X)$-modules over $C$. By construction we have the

Proposition 7.8 The Thom isomorphism $\phi$ induces an isomorphism

$$
E_{\mathbb{T}}(V) \cong E_{\mathbb{T}}(X)^{[\phi, V, \epsilon]}
$$

of $E_{\mathbb{T}}(X)$-modules. 
In the case of the orientation $\Sigma$ associated to the sigma function (4.13), we can be explicit about the open set on which

$$
\sigma\left(V^{a}, V^{b}, \epsilon\right) \stackrel{\text { def }}{=} e_{\Sigma}\left(V^{a}, V^{b}, \epsilon\right)
$$

is a unit.

Lemma 7.9 Let $\bar{B} \subset \mathbb{A}_{\text {an }}^{1}$ be the preimage of the ordinary points of $C$ : that is, the complement of the (closed) set of points $\bar{a} \in \mathbb{A}_{\text {an }}^{1}$ such that $X^{\wp(\bar{a})} \neq X^{\mathbb{T}}$ or $m \bar{a} \in \Lambda$ for $m$ a character of the action of $\mathbb{T}$ on $\left.V\right|_{X^{\mathbb{T}}}$. Then

$$
\sigma\left(V^{\mathbb{T}[n]}, V^{\mathbb{T}}, \epsilon\right) \in \mathcal{H}\left(X^{\mathbb{T}} ; \bar{B}\right)^{\times} .
$$

Proof Let $T$ be the standard maximal torus in $S O(2 d)$, giving an isomorphism

$$
\check{T} \cong \mathbb{Z}^{d} .
$$

The reduction $m$ is then an array or integers $m=\left(m_{1}, \ldots, m_{d}\right)$. It suffices to consider the case that

$$
\left.V\right|_{X^{\mathbb{T}}} \cong L_{1}+\cdots+L_{d}
$$

is a sum of line bundles, with $\mathbb{T}$ acting on $L_{i}$ by the character $m_{i}$. Let $x_{i}=$ $c_{1} L_{i}$. Then

$$
\begin{aligned}
\sigma\left(V^{\mathbb{T}[n]}, V^{\mathbb{T}}\right) & =\frac{\prod_{m_{j} \equiv 0(\bmod n)} \sigma\left(m_{j} z+x_{j}\right)}{\prod_{m_{j}=0} \sigma\left(m_{j} z+x_{j}\right)} \\
& =\prod_{0 \neq m_{j} \equiv 0} \sigma(\bmod n)
\end{aligned}
$$

Since the $x_{j}$ are nilpotent, this is a unit in a neighborhood of $z$ provided that $\prod_{0 \neq m_{j} \equiv 0(\bmod n)} \sigma\left(m_{j} z\right)$ is non-zero. This happens if and only if $m_{j} z \in \Lambda$.

As mentioned in the introduction, if f $W=\left(V_{0}, V_{1}\right)$ is a pair of $\mathbb{T}$-oriented vector bundles, then we extend the notation (7.1) by defining $E_{\mathbb{T}}(W)$ to be the invertible sheaf

$$
E_{\mathbb{T}}(W)=E_{\mathbb{T}}\left(V_{0}\right) \underset{E_{\mathbb{T}}(X)}{\otimes} E_{\mathbb{T}}\left(V_{1}\right)^{-1}
$$

of $E_{\mathbb{T}}(X)$-modules. As in Proposition 7.8, a choice of $\mathbb{T}$-orientation $\Omega$ on $W$ gives rise to a class $[\sigma, W, \Omega] \in H^{1}\left(C, E_{\mathbb{T}}(X)^{\times}\right)$, equipped with a canonical isomorphism of $E_{\mathbb{T}}(X)$-modules

$$
E_{\mathbb{T}}(W) \cong E_{\mathbb{T}}(X)^{[\sigma, W, \Omega]} .
$$




\section{A Thom class}

\subsection{Equivariant elliptic cohomology of principal bundles}

Suppose that $Q / X$ is a $\mathbb{T}$-equivariant principal $G$-bundle over $X$, so in particular $Q_{\mathbb{T}} / X_{\mathbb{T}}$ is a principal $G$-bundle as well. Applying $\hat{E}$ to the map

$$
X_{\mathbb{T}} \rightarrow B G
$$

classifying $Q_{\mathbb{T}} / X_{\mathbb{T}}$ induces a map

$$
\operatorname{sp} \hat{E}\left(X_{\mathbb{T}}\right) \stackrel{\text { sp } \hat{E}\left(Q_{\mathbb{T}}\right)}{\longrightarrow} \check{T} \otimes \widehat{C} / W
$$

as in (4.1).

If $F$ is a connected component of $X^{\mathbb{T}}$, and if

$$
m: \mathbb{T} \rightarrow T
$$

is a reduction of the action of $\mathbb{T}$ on $\left.Q\right|_{F}$, then Lemma 4.2 implies that the diagram

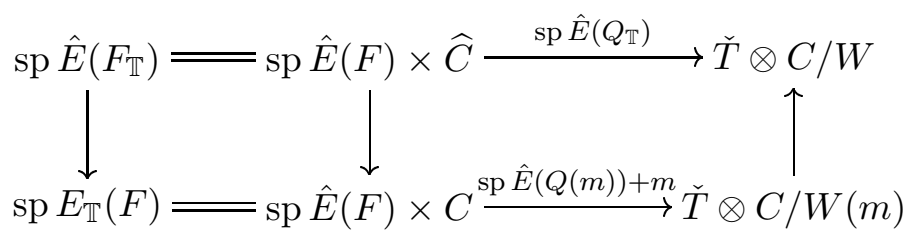

commutes. It follows that if we define

$$
\operatorname{sp} E_{\mathbb{T}}(Q(m)) \stackrel{\text { def }}{=} \operatorname{sp} \hat{E}(Q(m))+m,
$$

then we have the commutative solid diagram

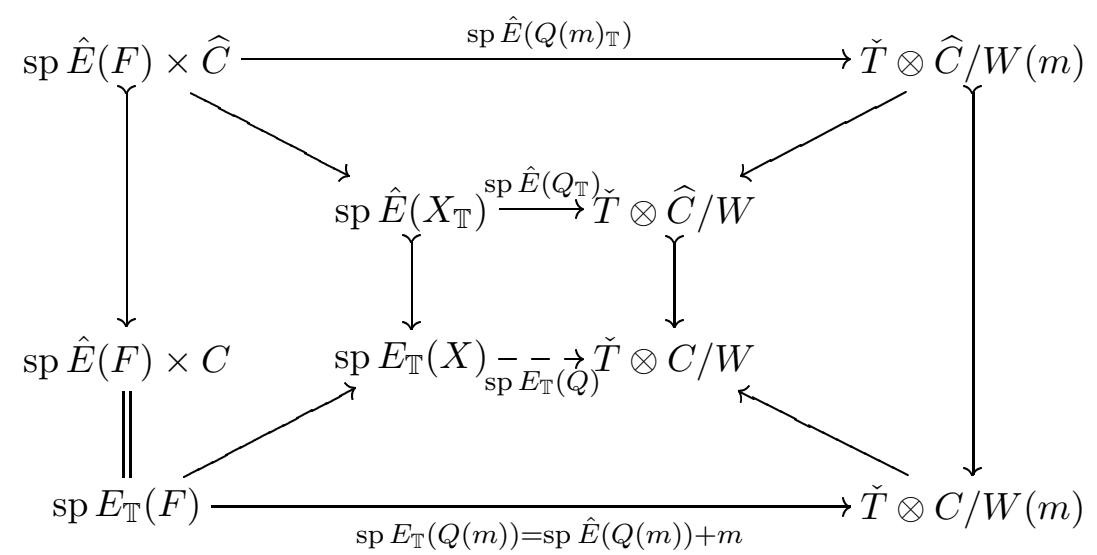


In writing this paper, we were guided by the idea (Conjecture 10.1) that there should be a canonical map sp $E_{\mathbb{T}}(Q)$ making the whole diagram (8.3) commute. We shall return to that question in section 10 , discussing both why such a map would be a good thing and why it is difficult to construct. For now we merely observe that the definition of $\operatorname{sp} E_{\mathbb{T}}(Q(m))$ implies the following.

As we have already observed before Lemma 4.2, the addition

$$
(\check{T} \otimes C) \times(\check{T} \otimes C) \rightarrow \check{T} \otimes C
$$

induces a translation

$$
(\check{T} \otimes C)^{W(m)} \times \check{T} \otimes C / W(m) \rightarrow \check{T} \otimes C / W(m),
$$

and so for $a \in C$ we get an operator

$$
\tau_{a^{m}}: \check{T} \otimes C / W(m) \rightarrow \check{T} \otimes C / W(m) .
$$

Lemma 8.4 For $a \in C$ the diagram

$$
\begin{array}{rc}
\operatorname{sp} \hat{E}(F) \times C \stackrel{\operatorname{sp} E_{\mathbb{T}}(Q(m))}{\longrightarrow} \check{T} \otimes C / W(m) \\
\operatorname{sp} \hat{E}(F) \times \tau_{a} \downarrow & \downarrow^{m} \\
\operatorname{sp} \hat{E}(F) \times C \stackrel{\operatorname{sp} E_{\mathbb{T}}(Q(m))}{\longrightarrow} \check{T} \otimes C / W(m)
\end{array}
$$

commutes.

\subsection{The Thom class}

Let $G$ be a spinor group, and let $G^{\prime}$ be a simple and simply connected compact Lie group, a unitary group, or indeed any compact connected Lie group with the property that the centralizer of any element is connected. Let $V$ be a $\mathbb{T}$ equivariant $G$-vector bundle over a finite $\mathbb{T}$-CW complex $X$, and let $V^{\prime}$ be a $\mathbb{T}$-equivariant $G^{\prime}$-bundle (by which we mean the vector bundle associated to a principal $G^{\prime}$ bundle via a linear representation of $G^{\prime}$ ). Suppose that $\xi^{\prime}$ is a degree-four characteristic classes for $G^{\prime}$, with the property that

$$
c_{2}\left(V_{\mathbb{T}}\right)=\xi^{\prime}\left(\left(V^{\prime}\right)_{\mathbb{T}}\right) .
$$

Suppose finally that $\theta^{\prime}$ is a theta function for $G^{\prime}$ of level $\xi^{\prime}$. In this section we prove the following 
Theorem 8.6 A $\mathbb{T}$-orientation $\epsilon$ on $V$ determines a canonical global section $\gamma=\gamma\left(V, V^{\prime}, \epsilon\right)$ of $E_{\mathbb{T}}(V)^{-1}$, such that

$$
\gamma_{0}=\theta^{\prime}\left(V_{\mathbb{T}}^{\prime}\right) \Sigma\left(V_{\mathbb{T}}\right)^{-1}
$$

under the isomorphism (6.6)

$$
\left(E_{\mathbb{T}}(V)^{-1}\right)_{0}^{\wedge} \cong \hat{E}\left(V_{\mathbb{T}}\right)^{-1}=\hat{H}\left(V_{\mathbb{T}}\right)^{-1}
$$

of $\hat{H}\left(X_{\mathbb{T}}\right)$-modules. The formation of $\gamma$ is natural in the sense if $f: Z \rightarrow X$ is a map of finite $\mathbb{T}-C W$ complexes, and

$$
\begin{aligned}
g / f: W / Z & \rightarrow V / X \\
g^{\prime} / f: W^{\prime} / Z & \rightarrow V^{\prime} / X
\end{aligned}
$$

are pull-backs of vector bundles, then under the isomorphism

$$
E_{\mathbb{T}}(g / f): E_{\mathbb{T}}(f)^{*} E_{\mathbb{T}}(V) \cong E_{\mathbb{T}}(W),
$$

we have

$$
E_{\mathbb{T}}(f)^{*} \gamma\left(V, V^{\prime}, \epsilon\right)=\gamma\left(W, W^{\prime}, g^{*} \epsilon\right)
$$

Let $\left\{U_{a}\right\}_{a \in C}$ be an indexed open cover of $C$ adapted to $(V, \Sigma, \epsilon)$ (Definition 7.5). By Proposition 7.8, to give a section of $E_{\mathbb{T}}(V)^{-1}$ satisfying (8.7) is equivalent to giving a global section of the sheaf $E_{\mathbb{T}}(X)^{-[\sigma, V, \epsilon]}$ whose value in

$$
\left(E_{\mathbb{T}}(X)^{-[\sigma, V, \epsilon]}\right)_{0}^{\wedge} \cong \hat{H}\left(X_{\mathbb{T}}\right)
$$

is $\theta^{\prime}\left(V_{\mathbb{T}}^{\prime}\right)$; this is what we shall do. The description of $E_{\mathbb{T}}(X)^{-[\sigma, V, \epsilon]}$ in section 7 shows that this amounts to giving sections $\gamma_{a} \in E_{\mathbb{T}, a}(X)\left(U_{a}\right)$ for $a \in C$, such that $\gamma_{0}=\theta^{\prime}\left(V_{\mathbb{T}}^{\prime}\right)$ and

$$
\psi_{a b}\left(\sigma(a, b, \epsilon) \gamma_{a}\right)=\gamma_{b}
$$

when $a$ is special and $b$ is ordinary.

Let $B \subset C$ be the set of ordinary points, and, as in Lemma 7.9, let $\bar{B} \subset \mathbb{A}_{\text {an }}^{1}$ be the preimage of $B$ in $\mathbb{A}_{\text {an }}^{1}$. Lemma 7.9 tells us that the formula

$$
\left.\bar{\gamma}_{B} \stackrel{\text { def }}{=} \theta^{\prime}\left(V_{\mathbb{T}}^{\prime}\right)\right|_{X^{\mathbb{T}}} \frac{\sigma\left(V^{\mathbb{T}}\right)}{\sigma\left(\left.V_{\mathbb{T}}\right|_{X^{\mathbb{T}}}\right)}
$$

defines an element of $\mathcal{H}\left(X^{\mathbb{T}} ; \bar{B}\right)$.

Lemma 8.9 For $\lambda \in \Lambda$,

$$
\tau_{\lambda}^{*} \bar{\gamma}_{B}=\bar{\gamma}_{B}
$$


Proof There is a $k$ such that

$$
q^{k}=e^{\lambda}
$$

Let $Q$ be the principal $G$-bundle associated to $V$, and let $Q^{\prime}$ be the principal bundle associated to $G^{\prime}$. If $F$ is a component of $X^{\mathbb{T}}$, let $m: \mathbb{T} \rightarrow T$ be a reduction of the action of $\mathbb{T}$ on $\left.Q\right|_{F}$, and let $m^{\prime}: \mathbb{T} \rightarrow T^{\prime}$ be a reduction of the action of $\mathbb{T}$ on $\left.Q^{\prime}\right|_{F}$.

The principal bundle $Q(m) / F$ is classified by a map

$$
F \stackrel{Q(m)}{\longrightarrow} B Z(m)
$$

in rational cohomology this becomes

$$
\operatorname{sp} H \mathbb{Q}(F) \stackrel{\text { sp } H \mathbb{Q}(Q(m))}{\longrightarrow} \operatorname{sp} H \mathbb{Q}(B Z(m)) \cong\left(\check{T} \otimes\left(\widehat{\mathbb{G}}_{a}\right)_{\mathbb{Q}}\right) / W(m),
$$

an sp $H \mathbb{Q}(F)$-valued point of $\left(\check{T} \otimes\left(\widehat{\mathbb{G}}_{a}\right)_{\mathbb{Q}}\right) / W(m)$. Since $F$ has the homotopy type of a finite CW-complex, the reduced cohomology of $F$ is nilpotent, and so we may consider $\exp (\operatorname{sp} H \mathbb{Q}(Q(m)))$ as an $\operatorname{sp} H \mathbb{Q}(F)$-valued point of $(\check{T} \otimes$ $\left.\left(\mathbb{G}_{m}\right)_{\mathbb{Q}}\right) / W(m)$.

Let

$$
\begin{aligned}
D & =\exp (\operatorname{sp} H \mathbb{Q}(Q(m))) \in\left(\check{T} \otimes \mathbb{G}_{m} / W(m)\right)(\operatorname{sp} H \mathbb{Q}(F)) \\
D^{\prime} & =\exp \left(\operatorname{sp} H \mathbb{Q}\left(Q^{\prime}\left(m^{\prime}\right)\right)\right) \in\left(\check{T} \otimes \mathbb{G}_{m}^{\prime} / W^{\prime}\left(m^{\prime}\right)\right)(\operatorname{sp} H \mathbb{Q}(F)) \\
u & =\exp (z) .
\end{aligned}
$$

Then

$$
\sigma\left(\left.V_{\mathbb{T}}\right|_{F}\right)(z)=\sigma_{d}\left(D u^{m}\right)
$$

and

$$
\begin{aligned}
\tau_{\lambda}^{*} \sigma\left(V_{\mathbb{T}}\right)(z) & =\sigma_{d}\left(D u^{m} q^{k m}\right) \\
& =\left(D u^{m}\right)^{-k \hat{I}(m)} q^{-k^{2} \phi(m)} \sigma\left(V_{\mathbb{T}}\right)(z) \\
& =D^{-k \hat{I}(m)} u^{-k I(m, m)} q^{-k^{2} \phi(m)} \sigma\left(V_{\mathbb{T}}\right)(z) .
\end{aligned}
$$

Similarly

$$
\tau_{\lambda}^{*} \theta^{\prime}\left(V_{\mathbb{T}}^{\prime}\right)(z)=\left(D^{\prime}\right)^{-k \hat{I}^{\prime}\left(m^{\prime}\right)} u^{-k I^{\prime}\left(m^{\prime}, m^{\prime}\right)} q^{-k^{2} \phi^{\prime}\left(m^{\prime}\right)} \theta^{\prime}\left(V_{\mathbb{T}}^{\prime}\right)(z) .
$$

If $c_{2}(V)_{\mathbb{T}}=\xi^{\prime}\left(V^{\prime}\right)_{\mathbb{T}}$, then Lemma 5.5 implies that

$$
\begin{aligned}
D^{\hat{I}(m)} & =\exp (\hat{I}(m)(\operatorname{sp} H \mathbb{Q}(Q(m))))=\exp \left(\hat{I}\left(m^{\prime}\right)\left(\operatorname{sp} H \mathbb{Q}\left(Q^{\prime}\left(m^{\prime}\right)\right)\right)\right)=\left(D^{\prime}\right)^{\hat{I}^{\prime}\left(m^{\prime}\right)} \\
\phi(m) & =\phi^{\prime}\left(m^{\prime}\right)
\end{aligned}
$$

which gives the result. 
Example 8.11 To illustrate the notation used in the proof, suppose we have chosen an isomorphism $T \cong \mathbb{T}^{d}$ and so also $\check{T} \cong \mathbb{Z}^{d}$. Then we have

$$
\check{T} \otimes \widehat{\mathbb{G}}_{a} \cong \widehat{\mathbb{A}}^{d} \cong \operatorname{spf} \mathbb{Z} \llbracket x_{1}, \ldots, x_{d} \rrbracket .
$$

Suppose that the map $F \rightarrow B G$ classifying $\left.V\right|_{F}$ factors through $B T$, i.e. that

$$
\left.V\right|_{F}=L_{1} \oplus \cdots \oplus L_{d}
$$

is written as a sum of complex line bundles. Then under the resulting map

$$
\operatorname{sp} H \mathbb{Q}(F) \rightarrow\left(\check{T} \otimes\left(\widehat{\mathbb{G}}_{a}\right)_{\mathbb{Q}}\right),
$$

the coordinate function $x_{j}$ pulls back to $c_{1} L_{j}$. If $\mathbb{T}$ acts on $L_{j}$ by the character $m_{j}$, then equation (8.10) becomes the more familiar equation

$$
\sigma\left(\left.V_{\mathbb{T}}\right|_{F}\right)(z)=\prod_{j} \sigma\left(e^{x_{j}+m_{j} z}\right) .
$$

Lemma 8.9 says that $\bar{\gamma}_{B}$ descends to a function $\gamma_{B}$ on

$$
\operatorname{sp} E\left(X^{\mathbb{T}}\right) \times B \subset \operatorname{sp} E\left(X^{\mathbb{T}}\right) \times C .
$$

For $b$ an ordinary point of $C$, we define

$$
\left.\gamma_{b} \stackrel{\text { def }}{=} \gamma_{B}\right|_{U_{b}} \in \mathcal{O}\left(\operatorname{sp} E\left(X^{b}\right) \times U_{b}\right)=E_{\mathbb{T}, b}(X)\left(U_{b}\right) .
$$

Now suppose that $a$ is a special point. Let $\bar{a}$ be a preimage of $a$ in $\mathbb{A}_{\text {an }}^{1}$, and define $\gamma_{a} \in E_{\mathbb{T}, a}(X)\left(U_{a}\right)$ by the formula

$$
\gamma_{a} \stackrel{\text { def }}{=} \tau_{-a}^{*}\left(\left(\left.\wp\right|_{W}\right)^{-1}\right)^{*}\left(R\left(V, V^{a}, \epsilon\left(V^{a}\right), \bar{a}\right) \theta^{\prime}\left(V^{\prime}, \bar{a}\right)\right),
$$

where $W \subset \wp^{-1} U_{a}$ is the component containing the origin (see (6.7)) and $R$ is the characteristic class defined in Corollary 5.29. This is a definition in view of the

Lemma 8.12 The class $\gamma_{a}$ is independent of the lift $\bar{a}$.

Proof Suppose that $\bar{a}$ and $\bar{a}^{\prime}$ are two lifts of $a$. Let $\alpha, B$, and $\delta$ be given by

$$
\begin{aligned}
& \alpha=\exp (\bar{a}) \\
& \beta=\exp \left(\bar{a}^{\prime}\right) \\
& \beta=\alpha q^{\delta} .
\end{aligned}
$$


Let $Y$ be a component of $X^{a}$. Let $m$ be a reduction of the action on $\mathbb{T}$ on $\left.Q\right|_{Y}$, and let $m^{\prime}$ be a reduction of the action of $\mathbb{T}$ on $\left.Q^{\prime}\right|_{Y}$. Lemma 5.25 implies that

$$
\begin{aligned}
\theta^{\prime}\left(Q^{\prime}, \bar{a}^{\prime}\right) & =w\left(a, q^{\frac{1}{n}}\right)^{\delta \phi(m)} \theta^{\prime}\left(Q^{\prime}, \bar{a}\right) \\
R\left(V, V^{a}, \epsilon\left(V^{a}\right), \bar{a}^{\prime}\right) & =w\left(a, q^{\frac{1}{n}}\right)^{-\delta \phi\left(m^{\prime}\right)} R\left(V, V^{a}, \epsilon\left(V^{a}\right), \bar{a}\right) .
\end{aligned}
$$

Equation (5.4) implies that

$$
\phi\left(m^{\prime}\right) \equiv \phi(m) \quad \bmod n
$$

which gives the result.

Lemma 8.13 The various sections $\gamma_{a}$ for $a \in C$ define a global section of $E_{\mathbb{T}}(X)^{-[\sigma, V]}$, whose value in $\left(E_{\mathbb{T}}(X)^{-[\sigma, V]}\right)_{0}^{\wedge}$ is $\theta^{\prime}\left(V^{\prime}\right)$.

Proof The value at 0 follows from the fact that

$$
R(V, V, 0)=1,
$$

as is easily checked. To see that the $\gamma_{a}$ assemble into a global section of $E_{\mathbb{T}}(X)^{-[\sigma, V]}$, we must show that, if $a$ is a special point of order $n$ and $b$ is ordinary with $U=U_{a} \cap U_{b}$ nonempty, then

$$
\psi_{a b}\left(\sigma(a, b, \epsilon) \gamma_{a b}\right)=\gamma_{b} .
$$

Let $i: X^{b} \rightarrow X^{a}$ be the inclusion. The diagram (6.9) defining $\psi_{a b}$ together with the equation (7.6) for $\sigma(a, b, \epsilon)$ reduces (8.14) to

$$
\tau_{b-a}^{*}\left(\sigma\left(V^{a}, V^{b}, \epsilon\right)^{-1} i^{*} \tau_{a}^{*} \gamma_{a}\right)=\tau_{b}^{*} \gamma_{b}
$$

or equivalently

$$
\sigma\left(V^{a}, V^{b}, \epsilon\right)^{-1} i^{*} \tau_{a}^{*} \gamma_{a}=\tau_{a}^{*} \gamma_{b} .
$$

Suppose that $Y$ is a component of $X^{a}$, and suppose that $F$ is a component of $X^{b}$ contained in $Y$. Let $\bar{m}$ be a reduction of the action of $\mathbb{T}$ on $\left.Q\right|_{F}$, and let $\bar{m}^{\prime}$ be a reduction of the action of $\mathbb{T}$ on $\left.Q^{\prime}\right|_{F}$. Lemma 5.5 and the equation $c_{2}\left(V_{\mathbb{T}}\right)=\xi^{\prime}\left(\left(V^{\prime}\right)_{\mathbb{T}}\right)$ imply that

$$
\begin{aligned}
\phi(\bar{m}) & =\phi^{\prime}\left(\bar{m}^{\prime}\right) \\
\hat{I}(\bar{m})(Q(\bar{m})) & =\hat{I}^{\prime}\left(\bar{m}^{\prime}\right)\left(Q^{\prime}\left(\bar{m}^{\prime}\right)\right) .
\end{aligned}
$$

As in the proof of Lemma 8.9, we let

$$
\begin{aligned}
D & =\exp (\operatorname{sp} H \mathbb{Q}(Q(\bar{m}))) \in\left(\check{T} \otimes \mathbb{G}_{m} / W(m)\right)(\operatorname{sp} H \mathbb{Q}(F)) \\
D^{\prime} & =\exp \left(\operatorname{sp} H \mathbb{Q}\left(Q^{\prime}\left(\bar{m}^{\prime}\right)\right)\right) \in\left(\check{T} \otimes \mathbb{G}_{m}^{\prime} / W^{\prime}\left(m^{\prime}\right)\right)(\operatorname{sp} H \mathbb{Q}(F)) \\
u & =\exp (z),
\end{aligned}
$$


and observe that Lemma 5.5 implies that

$$
\left(D u^{\bar{m}}\right)^{\hat{I}(\bar{m})}=\left(D^{\prime} u^{\bar{m}^{\prime}}\right)^{\hat{I}\left(\bar{m}^{\prime}\right)} .
$$

Let $\bar{a}$ be a preimage of $a$; let $k$ be the integer such that

$$
n \bar{a}=2 \pi i \ell+2 \pi i \tau k ;
$$

and let $\alpha=\exp (\bar{a})$.

By Lemma 3.9, $\left.\bar{m}\right|_{\mathbb{T}[n]}$ is a reduction of the action of $\mathbb{T}[n]$ on $\left.Q\right|_{Y}$ and similarly for $\bar{m}^{\prime}$. By Corollary 5.29 and Lemma 5.18, we may use $\bar{m}$ to calculate $R\left(V, V^{a}, \epsilon\left(V^{a}\right), \bar{a}\right)$ and $\bar{m}^{\prime}$ to calculate $\theta\left(V_{\mathbb{T}}^{\prime}, \bar{a}\right)$. The restriction to $F$ of the left side of (8.15) becomes

$$
\begin{aligned}
\left.\left.\frac{\sigma\left(V^{b}, \epsilon\right)}{\left.\sigma\left(V_{\mathbb{T}}^{a}, \epsilon\right)\right|_{F}} R\left(V, V^{a}, \epsilon, \bar{a}\right)\right|_{F} \theta^{\prime}\left(V_{\mathbb{T}}^{\prime}, \bar{a}\right)\right|_{F}= & \sigma\left(V^{b}, \epsilon\right) \\
& \frac{\left(D^{\prime} u^{\bar{m}^{\prime}}\right)^{\frac{k}{n} \hat{I}^{\prime}\left(\bar{m}^{\prime}\right)} \alpha^{\frac{k}{n} \phi^{\prime}\left(\bar{m}^{\prime}\right)}\left(\tau_{\bar{a}^{\prime}} \theta^{\prime}\right)\left(\left.V_{\mathbb{T}}^{\prime}\right|_{F}\right)}{\left(D u^{\bar{m}}\right)^{\frac{k}{n} \hat{I}(\bar{m})} \alpha^{\frac{k}{n} \phi(\bar{m})}\left(\tau_{\bar{a}^{\bar{m}}} \sigma\right)\left(\left.V_{\mathbb{T}}\right|_{F}\right)} \\
= & \frac{\sigma\left(V^{b}, \epsilon\right)}{\left(\tau_{\bar{a}} \sigma\right)\left(\left.V_{\mathbb{T}}\right|_{F}\right)}\left(\tau_{\bar{a}^{\bar{m}^{\prime}}} \theta^{\prime}\right)\left(\left.V_{\mathbb{T}}^{\prime}\right|_{F}\right) \\
= & \tau_{a}^{*}\left(\frac{\sigma\left(V^{b}, \epsilon\right)}{\sigma\left(\left.V_{\mathbb{T}}\right|_{F}\right)} \theta^{\prime}\left(\left.V_{\mathbb{T}}^{\prime}\right|_{F}\right)\right) \\
& =\tau_{a}^{*} \gamma_{b .}
\end{aligned}
$$

In the second equation we have equations (8.16) and (8.17). In the third equation we have used Lemma 8.4 and the fact that $\sigma\left(\left(V^{b}\right)_{\mathbb{T}}\right)=\sigma\left(V^{\mathbb{T}}\right)$ is invariant under translation.

This completes the construction of the section $\gamma$ promised in Theorem 8.6. The naturality (8.8) is straightforward, given the canonical nature of the sections $\gamma_{a}$.

\section{The sigma orientation}

Now suppose that $V=\left(V_{0}, V_{1}\right)$ is a pair of $\mathbb{T}$-equivariant spin vector bundles of even rank over a finite $\mathbb{T}$-CW complex $X$, with the property that

$$
c_{2}\left(V_{\mathbb{T}}\right)=0 .
$$

Recall ([6, 7]; see Lemma 5.27) that each $V_{i}$ is $\mathbb{T}$-orientable. 
Theorem 9.1 A $\mathbb{T}$-orientation $\epsilon$ on $V_{0}$ and $V_{1}$ determines a canonical trivialization $\gamma(V)=\gamma(V, \epsilon)$ of $E_{\mathbb{T}}(V)$, whose value in $E_{\mathbb{T}}(V)_{0}^{\wedge} \cong \hat{H}\left(\left(X_{\mathbb{T}}\right)^{V_{\mathbb{T}}}\right)$ is $\Sigma\left(V_{\mathbb{T}}\right)$ (see Definition 4.13). The association $V \mapsto \gamma(V)$ is stable, natural, and exponential in the sense described in Theorem 1.10.

Proof The proof proceeds much as the proof of Theorem 8.6: to construct $\gamma(V)$ it is equivalent to give a section $\gamma$ of $E_{\mathbb{T}}(X)^{[\sigma, V, \epsilon]}$ whose value in

$$
\left(E_{\mathbb{T}}(X)^{[\sigma, V, \epsilon]}\right)_{0}^{\wedge} \cong \hat{H}\left(X_{\mathbb{T}}\right)
$$

is 1 .

Once again, let $B \subset C$ be the set of ordinary points, and, as in Lemma 7.9, let $\bar{B} \subset \mathbb{A}_{\text {an }}^{1}$ be the preimage of $B$ in $\mathbb{A}_{\text {an }}^{1}$. Lemma 7.9 tells us that the formula

$$
\bar{\gamma}_{B} \stackrel{\text { def }}{=} \frac{\sigma\left(\left(V_{0}\right)_{\mathbb{T}}\right)}{\sigma\left(V_{0}^{\mathbb{T}}\right)} \frac{\sigma\left(V_{1}^{\mathbb{T}}\right)}{\sigma\left(\left(V_{1}\right)_{\mathbb{T}}\right)}
$$

defines an unit in $\mathcal{H}\left(X^{\mathbb{T}} ; \bar{B}\right)^{\times}$. The same argument as in Lemma 8.9 shows once again that $\bar{\gamma}_{B}$ descends to a function $\gamma_{B}$ on

$$
\operatorname{sp} E\left(X^{\mathbb{T}}\right) \times B \subset \operatorname{sp} E\left(X^{\mathbb{T}}\right) \times C .
$$

For $b$ an ordinary point of $C$, we define

$$
\left.\gamma_{b} \stackrel{\text { def }}{=} \gamma_{B}\right|_{U_{b}} \in \mathcal{O}\left(\operatorname{sp} E\left(X^{b}\right) \times U_{b}\right)^{\times}=E_{\mathbb{T}, b}(X)\left(U_{b}\right)^{\times} .
$$

Now suppose that $a$ is a special point. Let $\bar{a}$ be a preimage of $a$ in $\mathbb{A}_{\text {an }}^{1}$, and define $\gamma_{a}$ by the formula

$$
\left(\left.\wp\right|_{W}\right)^{*} \tau_{a}^{*} \gamma_{a} \stackrel{\text { def }}{=} \frac{R\left(V_{1}, V_{1}^{a}, \epsilon\left(V_{1}^{a}\right), \bar{a}\right)}{R\left(V_{0}, V_{0}^{a}, \epsilon\left(V_{0}^{a}\right), \bar{a}\right)}
$$

where $W \subset \wp^{-1}\left(U_{a}\right)$ is the component containing the origin, and $R$ is the characteristic class defined in Corollary 5.29. As in Lemma 8.12, $\gamma_{a}$ is independent of the lift $\bar{a}$. In this case, additionally, Corollary 5.29 implies that $\gamma_{a}$ is a unit, i.e. an element of $E_{\mathbb{T}, a}(X)\left(U_{a}\right)^{\times}$.

The same argument as in the proof of Lemma 8.13 shows that the sections $\gamma_{a}$ for $a \in C$ assemble into a global section of $E_{\mathbb{T}}(X)^{[\sigma, V, \epsilon]}$, which is a trivialization because it is so on each $U_{a}$.

The fact that the section $\gamma(V)$ is independent of the choice of $V_{i}$, as well as the fact that $\gamma\left(V \oplus V^{\prime}\right)=\gamma(V) \otimes \gamma\left(V^{\prime}\right)$ follows from definition of $\gamma(V)$ and the equation

$$
\sigma\left(V \oplus V^{\prime}\right)=\sigma(V) \sigma\left(V^{\prime}\right) .
$$

The naturality under change of base is clear from the construction. 


\section{A conceptual construction of the equivariant sigma orientation}

This section is devoted to a discussion of the following elaboration of Conjecture 1.14 .

Conjecture 10.1 Equivariant elliptic cohomology ought to have the following feature. Suppose that $Q$ is a $\mathbb{T}$-equivariant principal $G$-bundle over a $\mathbb{T}$-space $X$. Then there is a canonical map

$$
\operatorname{sp} E_{\mathbb{T}}(X) \stackrel{\operatorname{sp} E_{\mathbb{T}}(Q)}{\longrightarrow}(\check{T} \otimes C) / W
$$

making the diagram

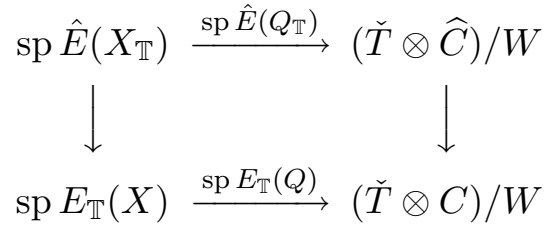

commute and having all the properties listed in Conjecture 1.14. Moreover, for all components $F$ of $X^{\mathbb{T}}$ and all reductions

$$
m: \mathbb{T} \rightarrow T
$$

of the action of $\mathbb{T}$ on $\left.Q\right|_{F}$, the diagram (8.3) should commute.

\subsection{Why the conjecture should be true, and why it is never- theless difficult to prove}

As explained in the introduction, the conjecture gives an elegant description of the equivariant sigma orientation, which even illuminates the non-equivariant case.

Although we have stated a conjecture, it is really a proposal of structure which should be sought in some equivariant elliptic cohomology theory. How difficult it is to establish the conjecture depends on your ontology. Given a fully developed theory of equivariant elliptic cohomology as proposed by GinzburgKapranov-Vasserot, it is not difficult at least to construct the map $\operatorname{sp} E_{\mathbb{T}}(Q)$.

To see this, recall that Ginzburg, Kapranov, and Vasserot have proposed that equivariant elliptic cohomology for the curve $C$ and the (compact connected Lie) group $G$ should be a covariant functor

$$
G K V_{G}(-):(G \text {-spaces }) \rightarrow(\text { schemes over }(\check{T} \otimes C) / W) .
$$


If $Q$ is a $\mathbb{T}$-equivariant principal $G$-bundle over $X$, then the $(\mathbb{T} \times G)$-equivariant elliptic cohomology of $Q$ should be a scheme

$$
G K V_{\mathbb{T} \times G}(Q) \rightarrow C \times(\check{T} \otimes C / W)
$$

over both $C$ (via the $\mathbb{T}$-action) and $\check{T} \otimes C / W$ (via the $G$-action). Now $G$ acts freely on $Q$ with quotient $X$, so one expects that

$$
G K V_{\mathbb{T} \times G}(Q) \cong G K V_{\mathbb{T}}(X) .
$$

Combining these observations leads to the prediction that a $\mathbb{T}$-equivariant principal $G$-bundle over $X$ should give rise to the map

$$
G K V_{\mathbb{T}}(X) \stackrel{G K V_{\mathbb{T}}(Q)}{\longrightarrow}(\check{T} \otimes C) / W .
$$

There are two problems with this proposal. First, we have in this paper described only $\mathbb{T}$-equivariant elliptic cohomology. It may not be difficult to resolve this problem: it is not so difficult to imagine an analogous construction of $G$ equivariant elliptic cohomology, and indeed Grojnowski does so in [11]. That leaves the second problem, that the underlying space of Grojnowski's functor is always $C$ : if

$$
\pi: G K V_{\mathbb{T}}(X) \rightarrow C
$$

is the structural map associated to an elliptic cohomology which is proposed by Ginzburg-Kapranov-Vasserot, then we have worked in this paper with the sheaf

$$
E_{\mathbb{T}}(X)=\pi_{*} \mathcal{O}_{G K V_{\mathbb{T}}(X)} .
$$

To see why this is a problem, note that in order to construct a map

$$
\operatorname{sp} E_{\mathbb{T}}(Q): \operatorname{sp} E_{\mathbb{T}}(X) \rightarrow \check{T} \otimes C / W
$$

we must in particular construct a map of topological spaces

$$
C \rightarrow(\check{T} \otimes C) / W .
$$

If $X^{\mathbb{T}}$ is non-empty, then $\operatorname{sp} E_{\mathbb{T}}\left(X^{T}\right)=X^{\mathbb{T}} \times C$, and for each connected component $F$ of $X^{\mathbb{T}}$, a reduction of the action of $\mathbb{T}$ on $\left.Q\right|_{F}$ gives a map

$$
m: C \rightarrow \check{T} \otimes C
$$

and so one has a place to start. But it is perfectly possible that $X^{\mathbb{T}}$ is empty, and then it is not clear how to proceed. For example, since

$$
\operatorname{sp} \hat{E}((B(\mathbb{Z} / N))) \cong \widehat{C}[N],
$$

one expects that

$$
G K V_{\mathbb{T}}(\mathbb{T} / \mathbb{T}[N]) \cong G K V_{\mathbb{T}[N]}(*) \cong C[N] .
$$


We shall cite three facts to support our conjecture. First, if $F \subseteq X^{\mathbb{T}}$ is a component of the fixed set, and if

$$
m: \mathbb{T} \rightarrow T
$$

is a reduction of the action of $\mathbb{T}$ on $F$, then using the map of diagram (8.3)

$$
\operatorname{sp} E_{\mathbb{T}}(Q(m))=\operatorname{sp} \hat{E}(Q(m))+m: \operatorname{sp} E_{\mathbb{T}}(F) \rightarrow \check{T} \otimes C / W(m) \rightarrow \check{T} \otimes C / W,
$$

it is easy to check that

$$
\left.\left.\mathcal{A}(V)\right|_{\mathrm{sp} E_{\mathbb{T}}\left(X^{\mathbb{T}}\right)} \cong \mathcal{A}\left(V^{\prime}\right)\right|_{\mathrm{sp} E_{\mathbb{T}}\left(X^{\mathbb{T}}\right)}
$$

when

$$
c_{2}\left(V_{\mathbb{T}}\right) \cong c_{2}\left(V_{\mathbb{T}}^{\prime}\right):
$$

this is the content of the proof of Lemma 8.9.

Second, the isomorphism

$$
E_{\mathbb{T}}(V) \cong \mathcal{I}(V)
$$

of the conjecture corresponds to the fact that the sigma function gives a trivialization of $E_{\mathbb{T}}(V)$ in Theorem 9.1.

Finally, we construct a map $\mathcal{E}(Q)$ for a stylized functor $\mathcal{E}$, which is not quite Grojnowski's elliptic cohomology, but captures its behavior on stalks. We simply throw away the points of $C$ for which we have no instructions for constructing a map (10.2). The functor $\mathcal{E}$ is inspired by the rational $\mathbb{T}$-equivariant elliptic spectra of Greenlees [10] and by Hopkins's study of characters and elliptic cohomology [12].

Recall that $\mathcal{R}$ denotes the category of ringed spaces. Let $\mathcal{S}$ be the category in which the objects are ringed spaces $\left(X, \mathcal{O}_{X}\right)$, and in which a map

$$
f=\left(f_{1}, f_{2}\right):\left(X, \mathcal{O}_{X}\right) \rightarrow\left(Y, \mathcal{O}_{Y}\right)
$$

is a map of spaces

$$
f_{1}: X \rightarrow Y
$$

and a map of sheaves of algebras over $X$

$$
f_{2}: \mathcal{O}_{X} \rightarrow f_{1}^{-1} \mathcal{O}_{Y}
$$

Let $S u b(\mathbb{T})$ be the category of closed subgroups of $\mathbb{T}$ with morphisms given by inclusions. We shall define $\mathcal{E}$ to be a functor

$$
\mathcal{E}:(\mathbb{T} \text {-spaces }) \rightarrow \mathcal{S}^{S u b(\mathbb{T})}
$$

from $\mathbb{T}$-spaces to the category of $S u b(\mathbb{T})$-diagrams in $\mathcal{S}$. 
Let $X$ be a $\mathbb{T}$-space. The ringed space $\mathcal{E}(X)(\mathbb{T})$ is just

$$
\mathcal{E}(X)(\mathbb{T})=\operatorname{sp} E\left(X^{\mathbb{T}}\right) \times C
$$

(which is empty if $X^{\mathbb{T}}$ is). If $\mathbb{T}[N] \subset \mathbb{T}$ is a finite subgroup and $X^{\mathbb{T}[n]}$ is empty, then

$$
\mathcal{E}(X)(\mathbb{T}[N])=\emptyset .
$$

Otherwise,

$$
\mathcal{E}(X)(\mathbb{T}[N])=C[N],
$$

with structure sheaf $j^{-1} E_{\mathbb{T}}(X)$, where $j$ denotes the inclusion

$$
j: C[N] \rightarrow C .
$$

Explicitly, for $U \subseteq C[N], \mathcal{E}(X)(\mathbb{T}[N])(U)$ is the product

$$
\mathcal{E}(X)(\mathbb{T}[N])(U)=\prod_{a \in U} E_{\mathbb{T}}(X)_{a}=\prod_{a \in U} E_{\mathbb{T}}\left(X^{A}\right)_{a},
$$

over $a \in U$ of the stalks of Grojnowski's elliptic cohomology. For $A=\mathbb{T}[N] \subseteq B$ with $X^{B}$ empty, the map

$$
\mathcal{E}(X)(A) \rightarrow \mathcal{E}(X)(B)
$$

is trivial; otherwise it is induced by the map of sheaves of algebras over $C$

$$
E_{\mathbb{T}}\left(\left(X^{A}\right)\right) \rightarrow E_{\mathbb{T}}\left(\left(X^{B}\right)\right)
$$

by restriction to $C[N]$.

Proposition 10.3 Let $G$ be a simple and simply connected Lie group, and let $Q$ be a principal $G$-bundle over a $\mathbb{T}$-space $X$. Then there is a canonical map

$$
\mathcal{E}(Q): \mathcal{E}(X) \rightarrow \check{T} \otimes C / W
$$

in $\mathcal{S}^{S u b(\mathbb{T})}$, where $\check{T} \otimes C / W$ is considered as a constant $S u b(\mathbb{T})$-diagram, such that the diagram

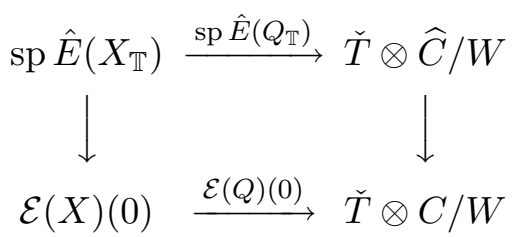

commutes, and such that for all components $F$ of $X^{\mathbb{T}}$ and all reductions

$$
m: \mathbb{T} \rightarrow T
$$

of the action of $\mathbb{T}$ on $\left.Q\right|_{F}$,

$$
\mathcal{E}\left(\left(\left.Q\right|_{F}\right)\right)(\mathbb{T})=\operatorname{sp} E(Q(m))+m .
$$


The proof will occupy the rest of this section.

Let $A$ be a closed subgroup of $\mathbb{T}$. Let $Y$ be a connected component of $X^{A}$. A reduction

$$
m: A \rightarrow T
$$

of the action of $A$ on $\left.Q\right|_{Y}$ determines a map

$$
C(A) \stackrel{C \otimes m}{\longrightarrow}(\check{T} \otimes C)^{W(m)} ;
$$

as usual if $a \in C(A)$ we write $a^{m}$ for the resulting element of $(\check{T} \otimes C)^{W(m)}$. The addition

$$
\check{T} \otimes C \times \check{T} \otimes C \stackrel{+}{\longrightarrow} \check{T} \otimes C
$$

induces a translation

$$
(\check{T} \otimes C)^{W(m)} \times \check{T} \otimes C / W(m) \stackrel{+}{\rightarrow} \check{T} \otimes C / W(m),
$$

and so we get an operator

$$
\tau_{a^{m}}: \check{T} \otimes C / W(m) \rightarrow \check{T} \otimes C / W(m) .
$$

If $A=\mathbb{T}$, then we define

$$
\mathcal{E}(Q)(\mathbb{T})_{Y, m}=\operatorname{sp} E(Q(m))+m,
$$

as required by the Proposition. If $A$ is finite, then we define the map of ringed spaces $\mathcal{E}(Q)(A)_{Y, m, a}$ to be the composition

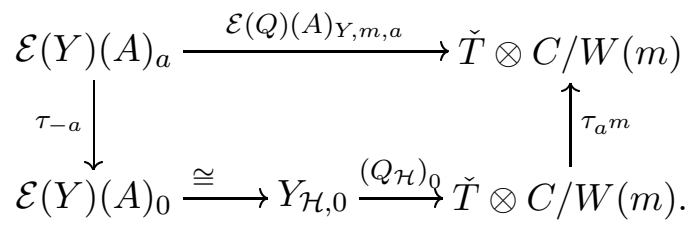

Here we have used the fact that the $\mathcal{E}(Y)(A)_{0}$ is just the origin in $C$, with ring

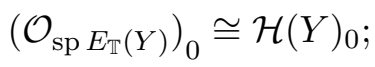

Lemma 4.3 provides the map $\left(Q_{\mathcal{H}}\right)_{0}$. We define

$$
\mathcal{E}(Q)(A)_{Y, m}=\coprod_{a \in C[N]} \mathcal{E}(Q)(A)_{Y, m, a}: \mathcal{E}(Y)(A) \rightarrow \check{T} \otimes C / W(m) .
$$

Lemma 10.4 If $m$ and $m^{\prime}$ are two reductions of the action of $A$ on $\left.Q\right|_{Y}$, then the diagram

commutes.

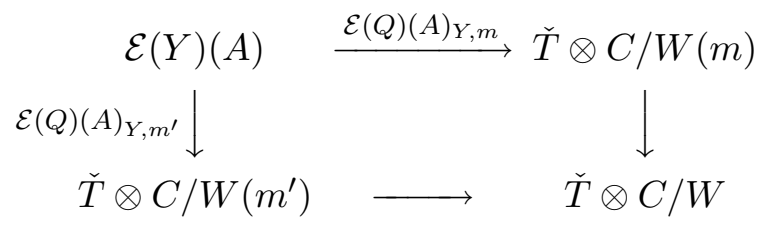


Proof This follows from the fact, proved in Lemma 3.6, that $m$ and $m^{\prime}$ differ by an element of the Weyl group of $G$.

The lemma permits us to write $\mathcal{E}(Q)(A)_{Y}$ for the map

$$
\mathcal{E}(Y)(A) \rightarrow \check{T} \otimes C / W
$$

described by (10.5). We define

$$
\mathcal{E}(Q)(A): \mathcal{E}(X)(A)=\coprod_{Y} \mathcal{E}(Y)(A) \stackrel{\amalg \mathcal{E}(Q)(A)_{Y}}{\longrightarrow} \check{T} \otimes C / W,
$$

where the coproduct is over the components $Y$ of $X^{A}$. The maps $\mathcal{E}(Q)(A)$ as $A$ ranges over closed subgroups of $\mathbb{T}$ assemble to give the map $\mathcal{E}(Q)$ of Proposition 10.3.

\subsection{Relationship to the theory $E_{\mathbb{T}}$}

The construction in Proposition 10.3 is closely related to the theory $E_{\mathbb{T}}$. We shall briefly explain how this works, as the explanation sheds light on the relationship between the "transfer argument" of Bott-Taubes and the geometry of the variety $(\check{T} \otimes C) / W$.

Suppose that $a$ is a special point of $C$ of order $N$ and let $A=\mathbb{T}[N]$. By definition we have a map

$$
\mathcal{E}(X)(A)_{a} \rightarrow \operatorname{sp} E_{\mathbb{T}, a}(X) ;
$$

indeed it is the inclusion of the stalk at $a$. Suppose that $b$ is an ordinary point, and let $U=U_{a} \cap U_{b}$. Suppose that $F$ is a component of $Y^{\mathbb{T}}$. Let

$$
m_{F}: \mathbb{T} \rightarrow T
$$

be a reduction of the action of $\mathbb{T}$ on $\left.Q\right|_{F}$; we write

$$
m_{Y}=\left.m_{F}\right|_{A}
$$

for the resulting reduction of the action of $A$ on $\left.Q\right|_{Y}$ as in Lemma 3.9. 
Consider the diagram:

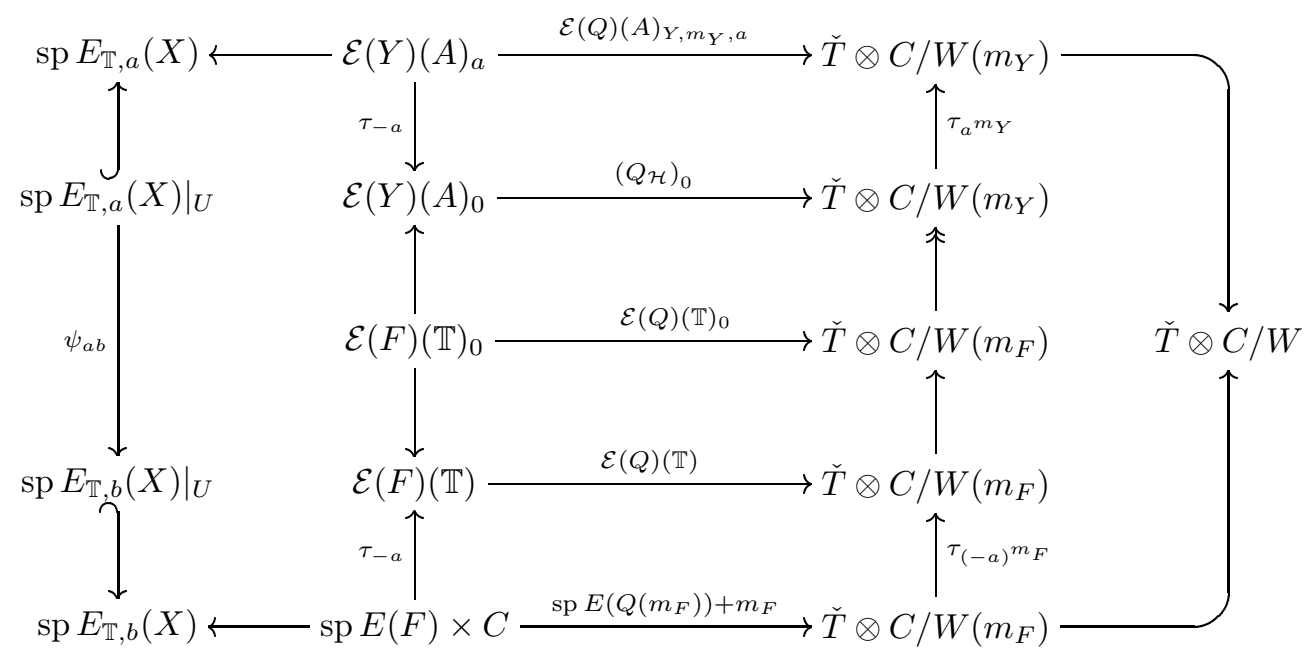

The commutativity of the rectangle on the left is just the definition of $\psi_{a b}$. The commutativity of the rectangle on the right is evident. The commutativity of the top and bottom rectangles in the middle is the definition of $\mathcal{E}(Q)$. The commutativity of the remaining rectangles in the middle follows from the group structure on $C$ and $\check{T} \otimes C$, together with the definitions of the maps involved.

Remark 10.7 We conclude this paper where the research for it began, with an explanation of the relationship between "transfer formula" of [7] and the diagram (10.6). Let $F \subseteq Y^{\mathbb{T}} \subseteq Y \subseteq X^{\mathbb{T}[N]}$ be as above. Let

$$
m \in \check{T}=\operatorname{hom}(\mathbb{T}, T)
$$

be a reduction of the action of $\mathbb{T}$ on $\left.Q\right|_{F}$ (so $m_{Y}=\left.m\right|_{\mathbb{T}[N]}$ is a reduction of the action of $\mathbb{T}[N]$ on $\left.\left.Q\right|_{Y}\right)$. Let $\theta \in \mathcal{O}\left(\check{T} \otimes \mathbb{G}_{a}^{\text {an }}\right)^{W}$ be a theta function for $G$; it determines a holomorphic characteristic class for principal $G$-bundles of the form $Q_{\mathbb{T}}$ : that is, the characteristic class $\theta\left(Q_{\mathbb{T}}\right)$ lies in $\mathcal{H}\left(X ; \mathbb{A}_{\text {an }}^{1}\right)$.

The first point is that, for any $a \in \mathbb{G}_{a}^{\text {an }}, \tau_{a^{m}} \theta \in \mathcal{O}\left(\check{T} \otimes \mathbb{G}_{a}^{\text {an }}\right)^{W(m)}$, so it gives a holomorphic characteristic class for principal $Z(m)$-bundles. Moreover, the commutativity of the diagram

$$
\begin{array}{rc}
\operatorname{sp} H(F) \times \mathbb{G}_{a}^{\mathrm{an}} \stackrel{\mathrm{sp} \mathcal{H}(Q(m))}{\longrightarrow} \check{T} \otimes \mathbb{G}_{a}^{\mathrm{an}} / W(m) \\
\left.\operatorname{sp} E(F) \times \tau_{a}\right\rfloor & \downarrow \tau_{a} \\
\operatorname{sp} H(F) \times \mathbb{G}_{a}^{\mathrm{an}} \stackrel{\operatorname{sp} \mathcal{H}(Q(m))}{\longrightarrow} \check{T} \otimes \mathbb{G}_{a}^{\mathrm{an}} / W(m)
\end{array}
$$


implies that

$$
\tau_{a}\left(\theta\left(\left.Q\right|_{F}\right)\right)=\left(\tau_{a^{m_{F}}} \theta\right)\left(Q\left(m_{F}\right)\right) \in \mathcal{H}\left(F ; \mathbb{A}_{\text {an }}^{1}\right) .
$$

The second point is that, if $a \in C[N]$, and $\bar{a}$ is a lift of $a$ to $\mathbb{A}_{\text {an }}^{1}$, then $\tau_{\bar{a}^{m}} \theta$ is nearly invariant under the action of $W\left(m_{Y}\right)$. Precisely, if $w \in W\left(m_{Y}\right)$, then

$$
\bar{a}^{w m}=\bar{a}^{m}+\lambda
$$

for some $\lambda \in \check{T} \otimes \Lambda$ : that is, $\bar{a}^{w m}$ and $\bar{a}^{m}$ are related by the action of the affine $W e y l$ group of $G$. Since $\theta$ is a theta function for $G$, the relationship between $\tau_{\bar{a}^{m}} \theta$ and $\tau_{\bar{a}^{w m}} \theta$ is controlled by $c_{2}\left(Q_{\mathbb{T}}\right)$ When this class is zero, or when the second Chern class of another bundle cancels it, then we may suppose that we have a characteristic class

$$
\left(\tau_{\bar{a}^{m_{Y}}} \theta\right)\left(Q\left(m_{Y}\right)\right) \in \mathcal{H}\left(Y ; \mathbb{A}_{\text {an }}^{1}\right) .
$$

We then have

$$
\left.\left(\tau_{a^{m}} \theta\right)\left(Q\left(m_{Y}\right)\right)\right|_{F}=\left(\tau_{a^{m}} \theta\right)(Q(m))=\tau_{a}\left(\theta\left(\left.Q\right|_{F}\right)\right),
$$

which is a typical "transfer formula".

\subsection{The nonequivariant case}

The conjecture is interesting already in the nonequivariant case. In order to compare with [2], we suppose that $V$ is an $S U(d)$-bundle over a space $X$. Let $T \subset S U(d)$ be the usual maximal torus, with Weyl group $W$. Let $C=\mathbb{C} / \Lambda$ be a complex elliptic curve, and let $E$ be the associated elliptic spectrum.

We then have a map

$$
X \rightarrow B S U(d)
$$

which in $E$-theory gives (by the splitting principle) a map

$$
X_{E} \stackrel{f}{\rightarrow} B S U(d)_{E} \cong(\check{T} \otimes \widehat{C}) / W .
$$

The line bundle $\mathcal{I}(V)=f^{*} \mathcal{I}\left(\sigma_{d}\right)$ is certainly canonically isomorphic to $V_{E}$ : this follows simply from the fact that the sigma function is of the form

$$
\sigma(z)=z+o\left(z^{2}\right) .
$$

Of course the line bundle $\mathcal{A}(V)$ is trivial when restricted to $\check{T} \otimes \widehat{C} / W$. But if $c_{2} V=0$, then $\mathcal{A}(V)$ has a canonical trivialization, since $\mathcal{A}(V)$ descends from the line bundle $\mathcal{L}\left(c_{2}\right)$ over $\check{T} \otimes C$ defined by

$$
\mathcal{L}\left(c_{2}\right)=\frac{\check{T} \otimes\left(\mathbb{G}_{m}^{\text {an }}\right) \times \mathbb{C}}{(u, \lambda) \sim\left(u q^{m}, u^{\hat{I}(m)} q^{\phi(m)} \lambda\right)}
$$

Geometry ${ }^{6}$ Topology, Volume 7 (2003) 
(see (5.8)). It follows that $\sigma(V)$ gives a trivialization of

$$
\mathcal{A}(V) \otimes \mathcal{I}(V) \cong V_{E} .
$$

Notice that we only needed $c_{2} V=0$ to get a trivialization of $\mathcal{A}(V)$ : this is because our elliptic curve is of the form $C=\mathbb{C} / \Lambda$, and the construction of $\mathcal{L}\left(c_{2}\right)$ uses the covering of $C$.

\section{References}

[1] Matthew Ando, Maria Basterra, The Witten genus and equivariant elliptic cohomology, Mathematische Zeitschrift, 240 (2002) 787-822

[2] Matthew Ando, Michael J Hopkins, Neil P Strickland, Elliptic spectra, the Witten genus, and the theorem of the cube, Inventiones Mathematicae, 146 (2001) 595-687, DOI 10.1007/s002220100175

[3] Matthew Ando, Power operations in elliptic cohomology and representations of loop groups Trans. Amer. Math. Soc. 352 (2000) 5619-5666

[4] Armand Borel, Topology of Lie groups and characteristic classes, Bull. Amer. Math. Soc. 61 (1955) 397-432

[5] Jean-Luc Brylinski, Representations of loop groups, Dirac operators on loop space, and modular forms, Topology, 29 (1990) 461-480

[6] Raoul Bott, Hans Samelson, Applications of the theory of Morse to symmetric spaces, Amer. J. Math. 80 (1958) 964-1029

[7] Raoul Bott, Clifford Taubes, On the rigidity theorems of Witten, J. Amer. Math. Soc. 2 (1989) 137-186

[8] Daniel S Freed, Edward Witten, Anomalies in string theory with D-branes, Asian J. Math. 3 (1999) 819-851

[9] V Ginzburg, M Kapranov, E Vasserot, Elliptic algebras and equivariant elliptic cohomology, preprint (1995)

[10] J P C Greenlees, Rational $S^{1}$-equivariant elliptic spectra, preprint (2001)

[11] Ian Grojnowski, Delocalized equivariant elliptic cohomology, unpublished manuscript (1994)

[12] Michael J Hopkins, Characters and elliptic cohomology, from: "Advances in Homotopy theory-Proc. James conference 1988", Cambridge University Press (1989)

[13] Michael J Hopkins, Topological modular forms, the Witten genus, and the theorem of the cube, Proceedings of the International Congress of Mathematicians, Vol. 1, 2 (Zürich, 1994), Birkhäuser, Basel (1995) 554-565

[14] Victor G Kac, Infinite dimensional Lie algebras, Cambridge University Press, second edition (1985) 
[15] Kefeng Liu, On modular invariance and rigidity theorems, J. Differential Geom. 41 (1995) 343-396

[16] Eduard Looijenga, Root systems and elliptic curves, Inventiones Math. 38 (1976) 17-32

[17] Andrew Pressley, Graeme Segal, Loop groups, Oxford Univ. Press (1986)

[18] Daniel Quillen, The spectrum of an equivariant cohomology ring. I, II, Ann. of Math. 94 (1971) 549-572 and 573-602

[19] Ioanid Rosu, Equivariant elliptic cohomology and rigidity, Amer. J. Math. 123 (2001) 647-677 